\title{
Harmonic Analysis of Linear Fields on the Nilgeometric Cosmological Model
}

\author{
Masayuki Tanimoto * \\ Max-Planck-Institut für Gravitationsphysik \\ (Albert-Einstein-Institut) \\ Am Mühlenberg 1, Golm 14476, Germany
}

June 4, 2018

\begin{abstract}
To analyze linear field equations on a locally homogeneous spacetime by means of separation of variables, it is necessary to set up appropriate harmonics according to its symmetry group. In this paper, the harmonics are presented for a spatially compactified Bianchi II cosmological model - the nilgeometric model. Based on the group structure of the Bianchi II group (also known as the Heisenberg group) and the compactified spatial topology, the irreducible differential regular representations and the multiplicity of each irreducible representation, as well as the explicit form of the harmonics are all completely determined. They are also extended to vector harmonics. It is demonstrated that the Klein-Gordon and Maxwell equations actually reduce to systems of ODEs, with an asymptotic solution for a special case.
\end{abstract}

\section{Introduction}

A basic strategy to analyze linear field equations on a given spacetime, like linear perturbation equations of Einstein's equation, is to separate the equations using appropriate harmonics. The harmonics for a given manifold are in general determined by the underlying symmetry group (isometry group) and the topology of the manifold. The simplest example is that of a commutative group acting on a flat manifold. If the manifold is compactified to, e.g., a torus, then functions on the manifold are expanded in the form of usual Fourier series. When the group is noncommutative, however, the harmonics become much more complicated. The most familiar example in homogeneous cosmology is the $\mathrm{SU}(2)$ (Bianchi IX) case [2, 8, 10, where one needs to use the spherical harmonics (and their generalization to vector and tensor harmonics if necessary) to separate field variables. A notable nontrivial example is the $H^{2} \times \mathbf{R}$ (Bianchi III) case [20, 16] with compactified three dimensional manifold. Since such a manifold is a direct product of two submanifolds, a closed hyperbolic plane and a circle, the harmonics are simply given by making products of those for the

*Email: masayuki.tanimoto@aei.mpg.de 
two lower dimensional manifolds. Note that the Bianchi III belongs to Class B 4]. Separations of variables regarding locally rotationally symmetric (LRS) Class A Bianchi types (and gravitational perturbations) were discussed in [3] 11 . without compactification.

In this paper we consider the generic (i.e., non-LRS) Bianchi II model with compactification. The Bianchi II type is one of the class A types. The underlying symmetry group is the 3 -dimensional Heisenberg group $H_{1}$, which we refer to as the Bianchi II group $G_{\text {II }}$. The Bianchi II homogeneous manifolds correspond to Thurston's nilgeometry [23]. We will refer to spatially compactified Bianchi II spacetimes (i.e., ones obtained from Bianchi II spatially homogeneous spacetimes by compactifying the homogeneous spatial sections) as nilgeometric cosmological models (to distinguish from the conventional open models).

By considering a compactified (spatial) manifold we have the following merits; (1) a compact Cauchy surface makes it natural to view the field equations as an initial value problem in cosmological context, (2) a finite spatial volume is physically reasonable, and (3) functional analysis on a compact manifold is much easier and tractable than one on an open manifold, since we can avoid many complexities involved in continuous spectra and those for the convergence of integrals (remember the contrast between Fourier series analysis on the "compactified space" $S^{1}=\mathbf{R}^{1} / \mathbf{Z}$ and Fourier analysis on the open space $\mathbf{R}$ ).

We also mention that a motivation of considering compactified manifolds also comes from recent evidences that the spatial topology and global dynamical properties of solutions of Einstein's equation are related in some ways. In particular, recent results [1, 5] suggest a very general picture of how an appropriately conformally transformed spatial manifold evolves in time by the vacuum Einstein equation, depending upon its topology. This picture motivates us to study linear perturbation equations for locally homogeneous solutions which have various spatial topologies.

Fortunately, although it is not as trivial as the torus compactification of a flat manifold, the compactification of Bianchi II manifolds (or Bianchi II type spacetimes) is not very difficult. We will describe our compactification following [12, 18, 19].

In this paper we explore detailed properties for the scalar and vector harmonics. We also demonstrate separation of variables for the Klein-Gordon scalar field equation and the source-free Maxwell equation. Although generalization to tensor harmonics is straightforward we leave their explicit presentation to a subsequent paper as well as a study of linear perturbations. This paper is intended to lay a solid basis for exploring those more complicated problems, or to be useful for many applications on the nilgeometric spacetime model like quantum field analysis.

Some of the mathematical background assumed in this paper and some related results obtained are the following. As well known (e.g.,[15]), the harmonics on a manifold $M$ on which a transformation group $G$ acts are naturally obtained through irreducible decomposition of a representation $T$, called the regular representation, of $G$ on $L^{2}(M)$. Let $g \in G$ and let $f(x) \in L^{2}(M)$. The (right) regular representation $\left(T, L^{2}(M)\right)$ is given by the homomorphism $T: g \rightarrow T_{g}$, where $T_{g}$ is the right translation map $T_{g}: f(x) \rightarrow f(x g)$. [25] In fact, letting $g, g^{\prime} \in G$, we can see $T_{g} T_{g^{\prime}} f(x)=\left(T_{g^{\prime}} f\right)(x g)=f\left(x g g^{\prime}\right)=T_{g g^{\prime}} f(x)$, showing $T$ is a representation (homomorphism), $T_{g} T_{g^{\prime}}=T_{g g^{\prime}}$. This representation is however not irreducible in general. The appropriate harmonics on $M$ are naturally 
obtained through an irreducible decomposition of $\left(T, L^{2}(M)\right)$.

In our context, the transformation group $G$ is the Bianchi II group $G_{\text {II }}$, which acts on $\tilde{M}=\mathbf{R}^{3}$ from the left simply transitively, i.e., for arbitrary $p, q \in \tilde{M}$ there exist a unique element $g \in G$ such that $g p=q$. Thanks to this property, choosing an arbitrary fixed point $o \in \tilde{M}$, e.g., the coordinate origin, one can identify the group $G_{\mathrm{II}}$ and the manifold $\tilde{M}, G_{\mathrm{II}}=\tilde{M}$, by associating $g o$ with $g$. With this identification, the manifold $\tilde{M}$ is also the group $G_{\mathrm{II}}$, and therefore the right action of $G_{\mathrm{II}}$ on $\tilde{M}$ is also naturally defined. We make $\tilde{M}$ compact by identifying points by left action of a discrete subgroup $A \subset G_{\mathrm{II}}, M=A \backslash \tilde{M}$. The (right) regular representation $\left(T, L^{2}(A \backslash \tilde{M})\right)$ on this space is indeed well defined, since keeping in mind the fact that we can identify an arbitrary function on $A \backslash \tilde{M}$ with an "automorphic function" $f(x)$ on $\tilde{M}$ such that $f(x)=f(A x)$, we can confirm the consistency $T_{g} f(A x)=f(A x g)=f(x g)=T_{g} f(x)$. This shows the consistency we choose the "right" regular representation, i.e., since we want to define the (Killing) symmetry of the manifold with respect to the left action, and make a quotient by the left one, the regular representation on the quotient should be the right one for commutativity. The universal covering manifold $\left(\tilde{M}, \tilde{q}^{(0)}\right)$ with a standard left invariant metric $\tilde{q}^{(0)}$ naturally defines a left invariant measure $d \mu_{0} \equiv d \mu_{\mathrm{L}}$ on $\tilde{M}$, for which we can define the natural inner product on $L^{2}(\tilde{M})$. Since for the Bianchi II group this measure is also right invariant $d \mu_{\mathrm{L}} \propto d \mu_{\mathrm{R}}$, i.e., $G_{\mathrm{II}}$ is unimodular, the right regular representation $\left(T, L^{2}(A \backslash \tilde{M})\right)$ with the inner product is unitary; $\int_{M}|f(x g)|^{2} d \mu_{0}=\int_{M}|f(x)|^{2} d \mu_{0}$.

Our mode functions on $M$ will be denoted as $\phi_{l, m, n_{0}}$ (or $\varphi_{l, m, n_{0}}$ on the spacetime $M \times \mathbf{R}$ ) for generic modes with $m \neq 0$. The index $m$ labels inequivalent irreducible representations, while the index $n_{0}$ labels ones in equivalent representations. For fixed $m$ and $n_{0}$, the functions $\left\{\phi_{l}\right\}_{l=0}^{\infty}$ work as a set of basis functions for the irreducible representation space specified by $m$ and $n_{0}$. Each single $\phi_{l}$ spans the eigenspace of an operator denoted as $L^{2}$, which is like a total angular momentum operator.

For the purpose of separation of variables, the most important relations are those for the differential representation, which is a linear transformation acting on the representation space spanned by mode functions. Those relations are written in terms of group-invariant differential operators denoted as $\chi_{I}(I=1 \sim 3)$. (See Eqs.(4.14) and (7.9).) Indeed, since group-invariant field equations like the Klein-Gordon equation can be written with no explicit coordinate dependences if it is written with the invariant operators, these relations are found to provide the key to separate the equations.

The formulas for the differential representation however do not provide complete information about the representation. For example, they do not tell whether the representation specified by $m$ does exist in $\left(T, L^{2}(M)\right)$, or how many copies of an equivalent representation exist in $\left(T, L^{2}(M)\right)$. To see how the representation $\left(T, L^{2}(M)\right)$ is decomposed to irreducible representations we need to find all the appropriate mode functions on $M$. In this paper the universal covering manifold $\tilde{M}$ (with a group invariant standard metric) is compactified to a circle bundle over the torus. The regular representation $\left(T, L^{2}(M)\right)$ is completely reducible, and as a result of finding of the mode functions on the given topology of $M$ we find the following:

Theorem 1.1 Let $M$ be the circle bundle over the torus with Euler class $e=1$ 
(see Eq. 2.15) for its fundamental group), and let $\left(T, L^{2}(M)\right)$ be the regular representation of the Bianchi II group $G_{\mathrm{II}}$. Then, it holds that

$$
T=\left(\bigoplus_{m \in \mathbf{Z} \backslash\{0\}}|m| T_{m}\right) \bigoplus\left(\bigoplus_{k_{1}, k_{2} \in \mathbf{Z}} \mathbf{1}_{k_{1}, k_{2}}\right),
$$

where $T_{m}$ is an infinite dimensional irreducible representation, $\mathbf{1}_{k_{1}, k_{2}}$ is a one dimensional irreducible representation, and the coefficient $|m|$ stands for the multiplicity in $T_{m} . \mathbf{Z} \backslash\{0\}$ represents nonzero integers.

(See [6], $\S 10$ and $\S 11$ and references therein for related mathematical works.) This decomposition expresses the completeness of the harmonics we construct. In particular, this decomposition does not depend on the Teichmüller (or moduli) parameters of $M$. From the Stone-von Neumann theorem [6] 22, $T_{m}$ is equivalent to a corresponding Schrödinger representation.

Construction of vector (or tensor) harmonics is not difficult on one hand. However the important point is to divide each irreducible space of vectors into subspaces such that each subspace is invariant under the action of the operator $L^{2}$. This feature is necessary to obtain decoupled systems of ODEs when the background spacetime has an additional symmetry. We define three kinds of vector harmonics, two of which have this property.

The plan of the paper is as follows. In the next section we describe the background solution and also account for some basic facts. Section 3 sets up some basic eigenvalues used to label mode functions, based on the compactification of the spatial manifold. In $\S$ we make algebraic discussions to derive the $\chi$-relations. Section 5 is devoted to construction of the mode functions on the spatial manifold. In section [6] the mode functions constructed on the spatial manifold are generalized to those on the spacetime. While $\S \S$ 田 6 deal with the generic modes, $\S \mathbf{Z}$ deals with the exceptional modes, the $U(1)$-symmetric modes, which complete all possible (scalar) modes. The results so far are applied to the Klein-Gordon equation and the reduced ODEs are explicitly given, with an asymptotic solution for a special case, in $\S$ In $\S 9$ we develop the vector harmonics. Section [10 is devoted to an application to Maxwell's equation. The final section is devoted to conclusion.

This paper is a full account, with much generalizations and development, of the subject outlined in $\S 3$ of 16]. Although most notations remain the same, one of the changes is that a quotient by the left action is now written $A \backslash \tilde{M}$ instead of $\tilde{M} / A$ to make clear which action is used. We employ the abstract index notation 24 and use leading Latin letters $a, b, \cdots$ to denote abstract indices for vectors and tensors in $\S \S 8]$ In the other sections however we write them without abstract indices. We often drop the tensor product symbol and write, e.g., $\left(\sigma^{1}\right)^{2}$ instead of $\sigma^{1} \otimes \sigma^{1}$. Beware that since vectors are also used as differential operators, products of them like $\left(\chi_{1}\right)^{2}$ can stand for second order derivatives like $\chi_{1} \chi_{1}$ or tensor products like $\chi_{1} \otimes \chi_{1}$, depending upon the quantity considered.

\section{The background solution}

Our background solution is specified by the following: (1) it is a solution of the vacuum Einstein equation, (2) it is spatially locally homogeneous of Bianchi II 
type, and (3) its spatial manifold is compact without boundary, in other words, closed. (An explicit topology will be chosen later.)

A Bianchi type II solution is characterized by the fact that the solution (or the universal cover of it) is invariant under the action of the Bianchi II group $G_{\text {II }}$, which is a three-dimensional nilpotent (e.g., 22]) Lie group. The group multiplication is given by

$$
(a, b, c)\left(a^{\prime}, b^{\prime}, c^{\prime}\right)=\left(a+a^{\prime}, b+b^{\prime}, c+c^{\prime}+a b^{\prime}\right),
$$

for $(a, b, c),\left(a^{\prime}, b^{\prime}, c^{\prime}\right) \in G_{\mathrm{II}}$. (Note: To save space we try to express components of group elements in a row form as above, but a column form is also equally used when it is more convenient.)

Let $\tilde{M}=\mathbf{R}^{3}$ be the simply-connected open manifold with coordinates $(x, y, z)$. We can define the group action on this manifold identifying the group manifold $G_{\text {II }}$ with $\tilde{M}$. The left-action is therefore expressed as

$$
(a, b, c)(x, y, z)=(a+x, b+y, c+z+a y),
$$

where $\mathbf{a}=(a, b, c) \in G_{\mathrm{II}}$, and $\mathbf{x}=(x, y, z) \in \tilde{M}\left(\simeq G_{\mathrm{II}}\right)$. Let $\xi_{I}(I=1,2,3)$ be the generators of the one-parameter subgroups $(a, 0,0),(0, b, 0)$ and $(0,0, c) \in$ $G_{\mathrm{II}}$. It is easy to find they are expressed

$$
\xi_{1}=\frac{\partial}{\partial x}+y \frac{\partial}{\partial z}, \quad \xi_{2}=\frac{\partial}{\partial y}, \quad \xi_{3}=\frac{\partial}{\partial z} .
$$

Similarly, the generators of the right actions and their dual one-forms are given by

$$
\begin{array}{r}
\chi_{1}=\frac{\partial}{\partial x}, \quad \chi_{2}=\frac{\partial}{\partial y}+x \frac{\partial}{\partial z}, \quad \chi_{3}=\frac{\partial}{\partial z} \\
\sigma^{1}=d x, \quad \sigma^{2}=d y, \quad \sigma^{3}=d z-x d y .
\end{array}
$$

These vectors $\chi_{I}$ and one-forms $\sigma^{I}(I=1 \sim 3)$ are called the invariant vectors or one-forms of $G_{\mathrm{II}}$, since they are left invariant;

$$
\mathcal{L}_{\xi_{I}} \chi_{J}=\left[\xi_{I}, \chi_{J}\right]=0=\mathcal{L}_{\xi_{I}} \sigma^{J}, \quad I, J=1 \sim 3,
$$

where $\mathcal{L}_{\xi_{I}}$ is the Lie derivative with respect to $\xi_{I}$. The invariant vectors satisfy the following commutation relations:

$$
\left[\chi_{1}, \chi_{2}\right]=\chi_{3}, \quad\left[\chi_{2}, \chi_{3}\right]=0, \quad\left[\chi_{3}, \chi_{1}\right]=0 .
$$

The vectors $\xi_{I}(I=1,2,3)$ are Killing vectors for the metric of the form $\tilde{q}=$ $\tilde{q}_{I J} \sigma^{I} \otimes \sigma^{J}$ with the components $\tilde{q}_{I J}$ being constants. Riemannian manifold $(\tilde{M}, \tilde{q})$ is called homogeneous, since $G_{\mathrm{II}}$ acts transitively on it as its isometry group.

A homogeneous metric is called locally rotationally symmetric (LRS) if it has a fourth independent Killing vector $\xi_{4}$. Bianchi type II LRS metrics are given by the metrics of the form $\tilde{q}^{(\mathrm{LRS})}=\tilde{q}_{11}\left(\left(\sigma^{1}\right)^{2}+\left(\sigma^{2}\right)^{2}\right)+\tilde{q}_{33}\left(\sigma^{3}\right)^{2}$, since such a metric has an additional Killing vector, given by

$$
\xi_{4}=-y \frac{\partial}{\partial x}+x \frac{\partial}{\partial y}+\frac{1}{2}\left(x^{2}-y^{2}\right) \frac{\partial}{\partial z} .
$$


This Killing vector generates the following one-parameter isometries $s_{\theta}=e^{\theta \xi_{4}}$ for the metric $\tilde{q}^{(\mathrm{LRS})}$ :

$$
s_{\theta}:\left(\begin{array}{l}
x \\
y \\
z
\end{array}\right) \rightarrow\left(\begin{array}{c}
R_{\theta}\left(\begin{array}{c}
x \\
y
\end{array}\right) \\
z+\zeta_{\theta}(x, y)
\end{array}\right),
$$

where $(x, y, z) \in \tilde{M}, R_{\theta}$ is the rotation matrix $R_{\theta}=\left(\begin{array}{cc}\cos \theta & -\sin \theta \\ \sin \theta & \cos \theta\end{array}\right)$, and

$$
\zeta_{\theta}(x, y) \equiv \frac{1}{2}\left(\left(x^{2}-y^{2}\right) \cos \theta-2 x y \sin \theta\right) \sin \theta .
$$

An LRS manifold $X=\left(\tilde{M}, \tilde{q}^{(\mathrm{LRS})}\right)$ has as a result a four dimensional isometry group Isom $X$. Let $\operatorname{Isom}_{0} X$ be its identity component, i.e., the component connected to the identity. An element $\alpha \in \operatorname{Isom}_{0} X$ can be uniquely expressed as the composite $\left(\alpha_{1}, \alpha_{2}, \alpha_{3}\right) \circ s_{\theta}$ for a choice of $\left(\alpha_{1}, \alpha_{2}, \alpha_{3}\right) \in G_{\text {II }}$ and $s_{\theta}$.

The one-parameter diffeomorphism $s_{\theta}$ plays an important role even when the metric is not LRS. It forms a one-parameter subgroup of the automorphism group of $G_{\mathrm{II}}$, which induces on the cotangent space a rotation of the invariant one-forms:

$$
s_{\theta *}:\left(\begin{array}{c}
\sigma^{1} \\
\sigma^{2} \\
\sigma^{3}
\end{array}\right) \rightarrow\left(\begin{array}{c}
R_{\theta}\left(\begin{array}{c}
\sigma^{1} \\
\sigma^{2}
\end{array}\right) \\
\sigma^{3}
\end{array}\right) .
$$

The induced map $s_{\theta}^{*}$ on tangent space acts on the invariant vectors $\chi_{I}$ the same way with replacement $R_{\theta} \rightarrow R_{-\theta}$ above. The significance of the automorphisms of Bianchi groups was first fully recognized by Jantzen 9 . Maps $s_{\theta *}$ or $s_{\theta}^{*}$ will be used in this paper in several contexts.

We can obtain the spacetime metric for the conventional Bianchi cosmology assuming that all the components with respect to the invariant frame $\left(d t, \sigma^{I}\right)$ formed by the invariant one-forms and the timelike basis $d t$ are functions of time $t$ only. The vacuum Bianchi II solution $\tilde{g}$ was first obtained by Taub [21]. We write that metric in the following form using our invariant one-forms (2.4):

$$
\tilde{\mathrm{g}}=-N^{2}(t) d t^{2}+q_{1}(t)\left(\sigma^{1}\right)^{2}+q_{2}(t)\left(\sigma^{2}\right)^{2}+q_{3}(t)\left(\sigma^{3}\right)^{2},
$$

where

$$
N^{2}=1+\beta^{2} t^{4 p_{3}}, \quad q_{1}=t^{2 p_{1}} N^{2}, \quad q_{2}=t^{2 p_{2}} N^{2}, \quad q_{3}=16 p_{3}^{2} \beta^{2} t^{2 p_{3}} / N^{2} .
$$

Parameters $p_{i}(i=1,2,3)$ and $\beta$ are constants such that $\beta>0, p_{3} \neq 0$, and

$$
\Sigma p_{i}=\Sigma p_{i}^{2}=1 .
$$

When $p_{1}=p_{2}$, the solution is LRS. Although there exist two possible such cases $\left(p_{1}, p_{2}, p_{3}\right)=(0,0,1)$ (case I LRS) and $(2 / 3,2 / 3,-1 / 3)$ (case II LRS), these two solutions represent equivalent one-parameter solutions. In fact, we can check that the case I LRS solution with $\beta=\beta_{\mathrm{I}}$ is isometric to the case II LRS solution with $\beta=\beta_{\mathrm{II}}=3^{-2 / 3} \beta_{\mathrm{I}}^{-1 / 3}$. When we are interested in an LRS solution, the case II LRS solution may be preferable, since the time coordinate $t$ in this solution approaches the proper time $\tau$ at future infinity. This will 
make comparisons with other models like the Bianchi type III [20, [16] more straightforward.

It is also worth pointing out that a solution $\tilde{\mathrm{g}}$ with $\left(p_{1}, p_{2}, p_{3}\right)$ is isometric to another solution $\tilde{\mathrm{g}}^{\prime}$ with $p_{1}$ and $p_{2}$ swapped. In fact, it is at once using Eq.(2.10) to see that $\tilde{\mathrm{g}}^{\prime}$ is the metric induced by $s_{\pi / 2} ; \tilde{\mathrm{g}}^{\prime}=s_{\pi / 2 *} \tilde{\mathrm{g}}$. We can therefore without loss of generality assume, e.g., $p_{1} \leq p_{2}$.

We denote the conventional solution described so far as $(\tilde{M} \times \mathbf{R}, \tilde{\mathrm{g}})$, and call it the universal covering solution. On the other hand our spatially closed solution, denoted $(M \times \mathbf{R}, \mathrm{g})$, is obtained introducing a spatial compactification with it. We express the solution as

$$
(M \times \mathbf{R}, \mathrm{g})=\Gamma \backslash(\tilde{M} \times \mathbf{R}, \tilde{\mathrm{g}}),
$$

using an appropriate discrete subgroup $\Gamma$ of $G_{\text {II }}$ which acts spatially from the left on the solution. The metric $\mathrm{g}$ here is the one induced from $\tilde{\mathrm{g}}$. ( $\mathrm{g}$ and $\tilde{\mathrm{g}}$ are therefore locally isometric to each other.) While there are infinitely many possible compactifications (i.e., spatial topologies), we for definiteness specify the spatial manifold $M$ to be "the circle bundle over the 2-torus with Euler class $e=1$." (See, e.g., [7, 14]. In general, a closed Bianchi II manifold is a Seifert fiber space over a Euclidean orbifold.) The fundamental group can be represented in the standard notation as

$$
\pi_{1}(M)=\left\langle g_{1}, g_{2}, g_{3} ;\left[g_{1}, g_{2}\right]=g_{3},\left[g_{1}, g_{3}\right]=1,\left[g_{2}, g_{3}\right]=1\right\rangle,
$$

where the brackets stand for group commutators, $[a, b] \equiv a b a^{-1} b^{-1}$. The procedure for the actual compactification is described in the next section. The resulting spatially compactified generalization was first constructed and discussed in 18. Note that as a result of the compactification the spatial manifold specified by $t=$ constant is now locally homogeneous (e.g., 14]), and the spacetime solution is said to be spatially locally homogeneous.

As shown in [18, $\Gamma$ contains four free parameters (see Eq.(6.1)). Our spatially closed solution (2.14) therefore forms a six parameter solution (since the universal cover has as we have seen two independent parameters, $\beta$ and, e.g., $\left.p_{3}\right)$.

\section{Compactification and eigenvalues}

To proceed, we need to describe the compactification of the spatial manifold and thereby define some eigenvalues.

Let us first describe the canonical way of expressing a Bianchi type II locally homogeneous manifold $(M, q)$. Let $\tilde{q}^{(0)}$ be the standard metric given by

$$
\tilde{q}^{(0)}=\left(\sigma^{1}\right)^{2}+\left(\sigma^{2}\right)^{2}+\left(\sigma^{3}\right)^{2}
$$

and let

$$
\mathcal{N} \equiv\left(\tilde{M}, e^{2 \alpha} \tilde{q}^{(0)}\right)
$$

be the standard conformal manifold, with $e^{2 \alpha}$ being a constant conformal factor. Then [12], the manifold $\left(M, q_{a b}\right)$ can be expressed as a quotient of such a standard conformal manifold (with an appropriate choice of the factor $e^{2 \alpha}$ ),

$$
(M, q)=A \backslash \mathcal{N},
$$


where $A$ is an appropriate discrete subgroup of the isometry group of $\mathcal{N}, A \subset$ $\operatorname{Isom} \mathcal{N}$

The subgroup $A$ must be isomorphic to the fundamental group $\pi_{1}(M)$ given by (2.15). (In fact, $M$ is a Haken manifold [7].) This means that $A$ must be an embedding of $\pi_{1}(M)$ into the isometry group $\operatorname{Isom\mathcal {N}}$. Let $\mathbf{a}_{i} \in \operatorname{Isom} \mathcal{N}$ ( $i=1,2,3)$ be the image of $\pi_{1}$-generator $g_{i}$ by such an embedding. Following the procedure shown in [12] [26], we find that it is possible to parameterize them in the following way:

$$
\mathbf{a}_{1}=(u, \delta, 0), \quad \mathbf{a}_{2}=(0,2 \pi v, 0), \quad \mathbf{a}_{3}=(0,0,2 \pi u v) .
$$

Here, $\mathbf{a}_{i} \in G_{\text {II }} \subset$ Isom $\mathcal{N}$. 227] We denote $A=\left\{\mathbf{a}_{1}, \mathbf{a}_{2}, \mathbf{a}_{3}\right\}$. The three real parameters $u, v$, and $\delta$ are called the Teichmüller parameters of geometric structure for the locally homogeneous 3-manifold $(M, q)$.

We construct the harmonics on $(M, q)$ by two steps; first we do the construction on a covering manifold, denoted $(\bar{M}, \bar{q})$, and then superpose appropriate subset of the harmonics to obtain those on $(M, q)$. The auxiliary manifold $(\bar{M}, \bar{q})$ is simply defined by removing a from $A$, i.e., $(\bar{M}, \bar{q})=\bar{A} \backslash \mathcal{N}$, where $\bar{A}=\left\{\mathbf{a}_{2}, \mathbf{a}_{3}\right\} . \bar{A}$ is a commutative subgroup of $A$, and therefore $\bar{M}$ is homeomorphic to the much tractable manifold $T^{2} \times \mathbf{R}$. In fact, we can easily see that it is each $x=$ constant plane in $\mathcal{N}$ that is compactified to a 2 -torus.

Now, let us introduce some important operators. We define the "total angular momentum-like" operator

$$
\mathrm{L}^{2} \equiv\left(\mathcal{L}_{\chi_{1}}\right)^{2}+\left(\mathcal{L}_{\chi_{2}}\right)^{2},
$$

using the Lie derivatives $\mathcal{L}_{\chi_{1}}$ and $\mathcal{L}_{\chi_{2}}$. Note that when acting on a scalar, it becomes a simple form

$$
\mathrm{L}^{2}=\left(\chi_{1}\right)^{2}+\left(\chi_{2}\right)^{2}
$$

which also coincides with the Laplacian $\triangle_{0}$ with respect to the standard metric $\tilde{q}^{(0)}$, up to square of $\chi_{3}$ :

$$
\triangle_{0}=\mathrm{L}^{2}+\left(\chi_{3}\right)^{2}
$$

when acting on a scalar. Here, $\chi_{I}(I=1,2,3)$ are regarded as differential operators. It is quite important to recognize that operators $\chi_{I}$ are well defined not only on the universal cover $\tilde{M}$ but also on the compactified manifold $M=A \backslash \tilde{M}$. In other words, the induced vector fields $\pi^{*} \chi_{I}$ on $M$ for the covering map $\pi: \tilde{M} \rightarrow M=A \backslash \tilde{M}$ is well defined because of the invariance of $\chi_{I}$ under the action of $A \subset G_{\mathrm{II}}$. (For simplicity we do not explicitly write $\pi^{*}$, and identify $\pi^{*} \chi_{I}$ and $\chi_{I}$.) Since $\bar{A}$ is also a subgroup of $G_{\mathrm{II}}, \chi_{I}$ are well defined on $\bar{M}$, also. The globally-defined invariant operators $\chi_{I}$ work as the fundamental derivative operators, since any group-invariant field equations become independent of coordinates when they are written with $\chi_{I}$. This coordinate-free property of the field equations is necessary to be able to reduce the field equation to ordinary differential equations.

The operator $\chi_{3}$, called the fiber generator, has a special importance, since it commutes with all the invariant operators $\chi_{I}$. In other words, $\chi_{3}$ is the center of the Bianchi II algebra. As a direct consequence of Schur's lemma, such an operator must be diagonalized to obtain an irreducible representation of the regular representation mentioned in Introduction. 
The operator $\mathrm{L}^{2}=\left(\chi_{1}\right)^{2}+\left(\chi_{2}\right)^{2}$ commutes with $\chi_{3}$, since $\chi_{3}$ is the center. We may therefore be able to diagonalize our mode functions with respect to both $\chi_{3}$ and $\mathrm{L}^{2}$. Also, consider another operator $\xi_{2}=\partial / \partial y$, which we can find commutes with both $\mathrm{L}^{2}$ and $\chi_{3}$, so we may diagonalize the mode functions with respect to $\xi_{2}$, also. This operator however is not well defined on $M$, but on $\bar{M}$. This is the reason we consider the auxiliary manifold $\bar{M}$. The existence of $\xi_{2}$ is important to make it possible to perform separation of variables for the eigenvalue equation for $\mathrm{L}^{2}$ (see below).

Let $i \mu$ and $i \nu$ be eigenvalues for the operators $\chi_{3}$ and $\xi_{2}$ :

$$
\chi_{3} \bar{\phi}=i \mu \bar{\phi}, \quad \xi_{2} \bar{\phi}=i \nu \bar{\phi} .
$$

The function $\bar{\phi}$ is supposed to be an appropriate mode function on $\bar{M}$. Also, we define $\lambda$ by $L^{2} \bar{\phi}=-\lambda^{2} \bar{\phi}$. The solution of these equations is given by

$$
\bar{\phi}=X(x) e^{i \mu z} e^{i \nu y},
$$

where the function $X(x)$ is a solution of the following "harmonic oscillator Schrödinger equation":

$$
\frac{d^{2} X}{d x^{2}}+\left(\lambda^{2}-(\mu x+\nu)^{2}\right) X=0 .
$$

For $\bar{\phi}$ to be well defined on $\bar{M}$, it must be an automorphic function such that $\bar{\phi}(\bar{A} \mathbf{x})=\bar{\phi}(\mathbf{x}) \cdot 28$. From this condition we find

$$
\begin{aligned}
& \mu=\mu(m)=m /(u v), \quad m \in \mathbf{Z}, \\
& \nu=\nu(n)=n / v, \quad n \in \mathbf{Z} .
\end{aligned}
$$

We call $\mu, m, \nu$, and $n$, respectively, the fiber eigenvalue, fiber index, auxiliary eigenvalue, and auxiliary index. We call $\lambda^{2}$ the total eigenvalue. The spectrum of $\lambda^{2}$ is determined in the next section.

\section{Irreducible differential representations}

It is not difficult to determine the irreducible representations of the regular representation in their differential form, i.e., the differential representations of the Bianchi II algebra. In fact, it will be found that this procedure is similar to the one in determining quantum states of the harmonic oscillator, since $L^{2}$ (or the scalar Laplacian $\triangle_{0}$ ) has essentially the same algebraic structure as that of the Hamiltonian of the oscillator.

As mentioned in the previous section since the fiber generator $\chi_{3}$ must be a constant when acting on an irreducible subspace, the fiber index $m \in \mathbf{Z}$ does not change values in this space. We therefore assume that $m$ is fixed throughout this section. It may be helpful to bear in mind that as for the correspondence to quantum mechanics, the fiber eigenvalue $\mu=m / u v$ corresponds to the Planck constant $h$, while $\chi_{1}$ and $\chi_{2}$ correspond, respectively, to the position $x$ and momentum $p$ operators, as in $[x, p]=i h \Leftrightarrow\left[\chi_{1}, \chi_{2}\right]=\chi_{3}=i \mu$. Remember however that our representation space is $L^{2}(M)$ instead of $L^{2}(\mathbf{R})$.

In this section we deal with the generic $m \neq 0$ case. The exceptional $m=0$ case will be discussed in $\S \mathbf{7}$ 
Let $\phi$ be an eigenfunction on $M$ for the operators $\chi_{3}$ and $L^{2}$, i.e., $\chi_{3} \phi=i \mu \phi$, and $\mathrm{L}^{2} \phi=-\lambda^{2} \phi$. It is helpful to introduce a symbol signifying the sign of the fiber eigenvalue, which allows us to discuss both $m \gtrless 0$ cases simultaneously; we define

$$
\varsigma \equiv \operatorname{sign}(m)=\operatorname{sign}(\mu) . \quad(m \neq 0)
$$

Let us then define

$$
\mathcal{A}_{1} \equiv \frac{1}{\sqrt{2}}\left(\chi_{1}+\varsigma i \chi_{2}\right), \quad \mathcal{A}_{2} \equiv \frac{1}{\sqrt{2}}\left(\chi_{1}-\varsigma i \chi_{2}\right), \quad \mathcal{A}_{3} \equiv-\varsigma i \chi_{3} .
$$

Then, we immediately find the following commutation relations

$$
\left[\mathrm{L}^{2}, \mathcal{A}_{1}\right]=\varsigma 2 i \mathcal{A}_{1} \chi_{3}, \quad\left[\mathrm{~L}^{2}, \mathcal{A}_{2}\right]=-\varsigma 2 i \mathcal{A}_{2} \chi_{3} .
$$

This means that $\mathcal{A}_{1}$ and $\mathcal{A}_{2}$ are, respectively, a raising and lowering operator for the total eigenvalue $\lambda^{2}$. In fact, since

$$
\mathrm{L}^{2} \mathcal{A}_{1} \phi=\left(\left[\mathrm{L}^{2}, \mathcal{A}_{1}\right]+\mathcal{A}_{1} \mathrm{~L}^{2}\right) \phi=\left(\varsigma 2 i \mathcal{A}_{1} \chi_{3}+\mathcal{A}_{1} \mathrm{~L}^{2}\right) \phi=-\left(2|\mu|+\lambda^{2}\right) \mathcal{A}_{1} \phi,
$$

$\mathcal{A}_{1} \phi$ is an eigenfunction for $\lambda^{\prime 2}=\lambda^{2}+2|\mu|$. Similarly, $\mathcal{A}_{2} \phi$ is an eigenfunction for $\lambda^{\prime 2}=\lambda^{2}-2|\mu|$.

Taking into account the fact that $\mathcal{A}_{1}$ and $\mathcal{A}_{2}$ change the eigenvalue $\lambda^{2}$ by $\pm 2|\mu(m)|$, we can without loss of generality assume the form of spectrum as

$$
\lambda^{2}=|\mu|\left(2 l+c_{m}\right),
$$

where

$$
l=0,1, \cdots .
$$

The value for $l=0, \lambda^{2}=\lambda_{0}^{2} \equiv|\mu| c_{m}$, corresponds to the smallest one for given $m$, which must exist because minus the Laplacian $-\triangle_{0}=-\mathrm{L}^{2}-\left(\chi_{3}\right)^{2}=$ $-\left(\mathrm{L}^{2}+\mu^{2}\right)$ can have only nonnegative eigenvalues. We call $l$ the spin index.

At this point we know the eigenmode is specified by the pair of integers $(l, m)$, so the corresponding eigenfunction can be expressed with these labels $\phi_{l, m}$. As we remarked since the value of $m$ does not change in an irreducible space, we drop $m$ and write $\phi_{l}$ for simplicity.

Let us write down the whole relations we have as

$$
\begin{aligned}
\mathrm{L}^{2} \phi_{l} & =-|\mu|\left(2 l+c_{m}\right) \phi_{l}, \\
\mathcal{A}_{3} \phi_{l} & =|\mu| \phi_{l}, \\
\mathcal{A}_{1} \phi_{l} & =\alpha_{l} \phi_{l+1}, \\
\mathcal{A}_{2} \phi_{l} & =\beta_{l} \phi_{l-1},
\end{aligned}
$$

where we have introduced unknown constants $\alpha_{l}$ and $\beta_{l}$, which possibly depend on $l$.

Note the following identity that can be easily checked by a direct computation:

$$
\mathrm{L}^{2}=2 \mathcal{A}_{1} \mathcal{A}_{2}-\mathcal{A}_{3} .
$$

Using Eqs.(4.7), this implies $-|\mu|\left(2 l+c_{m}\right)=2 \alpha_{l-1} \beta_{l}-|\mu|$, i.e.,

$$
\alpha_{l-1} \beta_{l}=-|\mu|\left(l+\frac{c_{m}-1}{2}\right) .
$$


Because of the arbitrariness of constant multipliers for the eigenfunctions $\phi_{l}$, we may set $\alpha_{l}$ or $\beta_{l}$ arbitrarily, but once it is set, the other is constrained from this relation. We take 29.

$$
\alpha_{l}=-\sqrt{|\mu|}, \quad \beta_{l}=\sqrt{|\mu|}\left(l+\frac{c_{m}-1}{2}\right) .
$$

These do satisfy Eq. (4.9). Then, since we defined $l$ so that $l=0$ gives, for given $m \neq 0$, the smallest eigenvalue of $-L^{2}$, we should have

$$
\mathcal{A}_{2} \phi_{0}=\beta_{0} \phi_{-1}=\sqrt{|\mu|}\left(\frac{c_{m}-1}{2}\right) \phi_{-1}=0,
$$

implying

$$
c_{m}=1
$$

So, now we have

$$
\alpha_{l}=-\sqrt{|\mu|}, \quad \beta_{l}=\sqrt{|\mu|} l .
$$

Gathering Eqs.(4.2), (4.7), and (4.13), we arrive at the following set of relations:

$$
\begin{aligned}
\chi_{1} \phi_{l} & =-\sqrt{\frac{|\mu|}{2}}\left(\phi_{l+1}-l \phi_{l-1}\right), \\
\chi_{2} \phi_{l} & =\varsigma i \sqrt{\frac{|\mu|}{2}}\left(\phi_{l+1}+l \phi_{l-1}\right), \\
\chi_{3} \phi_{l} & =i \mu \phi_{l}, \\
l & =0,1, \cdots .
\end{aligned}
$$

In particular,

$$
\mathrm{L}^{2} \phi_{l}=-\lambda_{l}^{2} \phi_{l}, \quad \lambda_{l}^{2} \equiv|\mu|(2 l+1) .
$$

Now, we have found the following. A "ground state" $\phi_{0}$ is determined as a solution for the two equations

$$
\mathcal{A}_{3} \phi_{0}=|\mu| \phi_{0}, \quad \mathcal{A}_{2} \phi_{0}=0 .
$$

Note that the function $\phi_{0}$ obtained this way is automatically an eigenfunction of $L^{2}$ as seen from the identity (4.8). The excited states $\phi_{l}$ are determined by successively multiplying the raising operator $(-1 / \sqrt{|\mu|}) \mathcal{A}_{1}$, i.e.,

$$
\phi_{l}=\left(-\frac{\mathcal{A}_{1}}{\sqrt{|\mu|}}\right)^{l} \phi_{0} .
$$

The space spanned by these functions, $L_{m}^{2}(M) \equiv\left\{\sum_{l=0}^{\infty} a_{l} \phi_{l} \mid a_{l} \in \mathbf{C}\right\} \cap L^{2}(M)$, gives an irreducible subspace of $L^{2}(M)$. In other words, the restriction of the regular representation $T$ to $L_{m}^{2}(M)$, denoted as $\left(T_{m}, L_{m}^{2}(M)\right)$, gives an irreducible representation. The differential representation $\left(d T_{m}, L_{m}^{2}(M)\right)$ is given by Eqs. (4.14), which will be repeatedly used to separate the field equations. For convenience, we call these relations the $\chi$-relations. 


\section{Mode functions on the compactified manifold}

In this section we solve the eigenvalue equations for the mode functions $\phi_{l}$ under the appropriate automorphic conditions. As a result we find how many equivalent copies of the irreducible representation $T_{m}$ are contained in $T$, in other words, the multiplicity of $T_{m}$ is determined. The explicit form of $\phi_{l}$ itself is also of great interest. In this section we continue to assume $m \neq 0$.

One of the possible procedures to find explicit form of $\phi_{l}$ is to solve the equations (4.16) to find $\phi_{0}$ and compute successive differential operations in Eq. (4.17) to find general $\phi_{l}$. Another procedure is to directly solve the eigenvalue equation $\mathrm{L}^{2} \phi_{l}=-\lambda^{2} \phi_{l}$ for general spin index $l$. While both ways are possible, we take the latter, which provides quicker way of identifying the solutions with known functions.

As remarked in $\S$ B let us find the mode functions on $\bar{M}$ first. Note that Eq.(3.10) becomes (attaching index $l$ to $X$ )

$$
\frac{d^{2} X_{l}}{d \zeta^{2}}+\left(l+\frac{1}{2}-\frac{\zeta^{2}}{4}\right) X_{l}=0
$$

if we define

$$
\zeta=\varsigma \sqrt{\frac{2}{|\mu|}}(\mu x+\nu)
$$

Independent solutions to the above equation are given by $D_{l}(\zeta)$ and $D_{-l-1}(i \zeta)$, where $D_{l}(\zeta)$ is the Weber parabolic cylinder function. When $l$ is zero or a positive integer, $D_{l}(\zeta)$ can be expressed using the Hermite polynomial $H_{l}(\zeta)$;

$$
D_{l}(\zeta)=e^{-\frac{1}{4} \zeta^{2}} H_{l}(\zeta)
$$

Our convention for the Hermite polynomial is $H_{l}(\zeta)=(-1)^{l} e^{\frac{1}{2} \zeta^{2}}\left(d^{l} / d \zeta^{l}\right) e^{-\frac{1}{2} \zeta^{2}}$.

Since $\phi_{0}$ must be annihilated by $\mathcal{A}_{2}$ the appropriate choice is found to be $D_{l}(\zeta)$, i.e., we must take $X_{l}=$ constant $\times D_{l}(\zeta)$. In fact, the equation $\mathcal{A}_{2} \bar{\phi}_{0}=0$ together with the separation form $\bar{\phi}_{l}=X_{l}(x) e^{i \nu y} e^{i \mu z}$, implies $(d / d \zeta+$ $(1 / 2) \zeta) X_{0}=0$, with the solution being $X_{0}=$ constant $\times e^{-(1 / 4) \zeta^{2}}$. This coincides with the one claimed for $l=0$. (Conversely, as we will see, functions $\phi_{l}$ constructed using these $X_{l}$ can satisfy the desired relations (4.14) for all $l$, which justifies our choice.)

The mode functions on $\bar{M}$ are therefore, attaching indices $m$ and $n$, given by

$$
\bar{\phi}_{l, m, n}(\mathbf{x})=C_{l} D_{l}\left( \pm \sqrt{\frac{2}{|\mu|}}(\mu x+\nu)\right) e^{i \mu z} e^{i \nu y}
$$

where $C_{l}$ are constants and $\mu$ and $\nu$ are defined in Eqs. (3.11).

The constants $C_{l}$ are determined by requiring that the functions $\bar{\phi}_{l, m, n}(\mathbf{x})$ obey the $\chi$-relations (4.14). Using the widely known formulas

$$
\begin{aligned}
D_{l}^{\prime}(\zeta) & =-\frac{1}{2}\left(D_{l+1}(\zeta)-l D_{l-1}(\zeta)\right), \\
\zeta D_{l}(\zeta) & =D_{l+1}(\zeta)+l D_{l-1}(\zeta),
\end{aligned}
$$

we can easily find $C_{l+1}=C_{l}$, i.e., they are constants that do not depend on $l$;

$$
C_{l}=C \text {. }
$$


(Actually, this is the reason we chose Eqs.4.13).) The constant $C$ may be determined by a normalization of the square integral on $M$. (See below.)

The mode functions on $M$ are, as mentioned, expressed as an infinite sum of these eigenfunctions on $\bar{M}$. Remember that they must be invariant under the action of $A=\left\{\mathbf{a}_{1}, \mathbf{a}_{2}, \mathbf{a}_{3}\right\}$, and the functions $\bar{\phi}_{l, m, n}$ are already invariant under $\bar{A}=\left\{\mathbf{a}_{2}, \mathbf{a}_{3}\right\}$. We therefore want to make a linear combination of $\bar{\phi}_{l, m, n}$ so that it is invariant under $\mathbf{a}_{1}$. Recalling the multiplication rule (2.2), we find the following transformation law (cf. [16, Eq.(3.15))

$$
\bar{\phi}_{l, m, n}\left(\mathbf{a}_{1} \mathbf{x}\right)=e^{i \frac{\delta}{v} n} \bar{\phi}_{l, m, n+m}(\mathbf{x}) .
$$

From this we can see that the following function $\phi_{l, m, n_{0}}$, defined as an infinite sum, is actually invariant under the action of $\mathbf{a}_{1}$ (cf. [16], Theorem 3.1), i.e., $\phi_{l, m, n_{0}}\left(\mathbf{a}_{1} \mathbf{x}\right)=\phi_{l, m, n_{0}}(\mathbf{x})$ for

$$
\phi_{l, m, n_{0}}(\mathbf{x})=\sum_{k=-\infty}^{\infty} e^{i \delta\left(n_{0} k+m \frac{k(k-1)}{2}\right)} \bar{\phi}_{l, m, n_{0}+m k}(\mathbf{x}),
$$

where $l=0,1, \cdots, \infty, \quad|m|=1,2, \cdots, \infty, \quad n_{0}=0,1, \cdots,|m|-1$. The sum is convergent at any point $\mathbf{x}$. It is easy to see that since the functions $\bar{\phi}_{l, m, n}(\mathbf{x})$ satisfy the relations (4.14), so do $\phi_{l, m, n_{0}}(\mathbf{x})$. These functions are therefore the right mode functions on $M=A \backslash \tilde{M}$.

As a result of the compactification, the index $n_{0}$ for the mode functions on $M$ is now bounded by $|m| .|m|$ is the multiplicity of the modes specified by the same $l$ and $m$. Since for each $n_{0}$ the functions $\phi_{l, m}$ span an irreducible subspace of $L^{2}(M),|m|$ is also the multiplicity of the irreducible representation $T_{m}$ contained in $T$.

We can summarize the results as follows.

Theorem 5.1 There exist $|m|$ different sets of mode functions $\left\{\phi_{l}(\mathbf{x})\right\}_{l=0}^{\infty}$ that satisfy the relations $\sqrt{4.14}$ ) on the compactified manifold $\left(M, q_{a b}\right)=A \backslash \mathcal{N}$ (with $M$ being the $S^{1}$-bundle over the 2-torus with Euler class $e=1$ ).

Let us discuss how the mode functions can be normalized. We define the inner product in $L^{2}(M)$ as

$$
(f, g) \equiv \int_{M} f g^{*} d \mu_{0}
$$

where $g^{*}$ is the complex conjugate of $g, d \mu_{0}=\sigma^{1} \wedge \sigma^{2} \wedge \sigma^{3}=d x d y d z$ is the standard invariant measure. We want to determine the square norm

$$
N_{l} \equiv\left(\phi_{l}, \phi_{l}\right) \text {. }
$$

We first observe the following.

Lemma 5.2 The invariant operators $\chi_{I}(I=1 \sim 3)$ in $L^{2}(M)$ are antiselfadjoint, $\chi_{I}^{\dagger}=-\chi_{I}$.

Proof. Since

$$
\left(\chi_{I} f, g\right)=\int_{M}\left(\chi_{I}\left(f g^{*}\right)-f \chi_{I} g^{*}\right) d \mu_{0}=\mathcal{I}_{I}-\left(f, \chi_{I} g\right),
$$


we need to show $\mathcal{I}_{I} \equiv \int_{M} \chi_{I}\left(f g^{*}\right) d \mu_{0}=0$. In fact, when, e.g., $I=1$, we can show $\mathcal{I}_{1}=\int_{M} \chi_{1}\left(f g^{*}\right) \sigma^{1} \wedge \sigma^{2} \wedge \sigma^{3}=\int_{M} d\left(f g^{*} \sigma^{2} \wedge \sigma^{3}\right)$, which is from Stoke's theorem $\int_{\partial M} f g^{*} \sigma^{2} \wedge \sigma^{3}=0$. Here, we have used the identity

$$
d f=\left(\chi_{1} f\right) \sigma^{1}+\left(\chi_{2} f\right) \sigma^{2}+\left(\chi_{3} f\right) \sigma^{3},
$$

which is valid for an arbitrary function $f$ on $M$, and also used the relation $d\left(\sigma^{2} \wedge \sigma^{3}\right)=0$, which is confirmed from the definition (2.4). The other cases $I=2,3$ are the same, since $d\left(\sigma^{1} \wedge \sigma^{3}\right)=d\left(\sigma^{1} \wedge \sigma^{2}\right)=0$.

REMARK. Operators $(1 / i) \chi_{I}(I=1 \sim 3)$ are selfadjoint.

Corollary 5.3 In $L^{2}(M), \mathcal{A}_{1}^{\dagger}=-\mathcal{A}_{2}$. $-\mathcal{A}_{2}$.

Proof. $\mathcal{A}_{1}^{\dagger}=2^{-1 / 2}\left(\chi_{1}+\varsigma i \chi_{2}\right)^{\dagger}=2^{-1 / 2}\left(\chi_{1}^{\dagger}-\varsigma i \chi_{2}^{\dagger}\right)=-2^{-1 / 2}\left(\chi_{1}-\varsigma i \chi_{2}\right)=$

Returning to the issue of $N_{l}$, consider $N_{l+1}=\left(\phi_{l+1}, \phi_{l+1}\right)$. When $m \neq 0$, from Eqs. (4.7) and Corollary [5.3 we have

$$
\begin{aligned}
N_{l+1} & =\frac{1}{\alpha_{l} \alpha_{l}^{*}}\left(\mathcal{A}_{1} \phi_{l}, \mathcal{A}_{1} \phi_{l}\right) \\
& =\frac{1}{\alpha_{l} \alpha_{l}^{*}}\left(\phi_{l}, \mathcal{A}_{1}^{\dagger} \mathcal{A}_{1} \phi_{l}\right) \\
& =\frac{-1}{\alpha_{l} \alpha_{l}^{*}}\left(\phi_{l}, \mathcal{A}_{2} \mathcal{A}_{1} \phi_{l}\right) \\
& =\frac{-1}{\alpha_{l} \alpha_{l}^{*}}\left(\phi_{l}, \beta_{l+1} \alpha_{l} \phi_{l}\right) \\
& =\frac{-\beta_{l+1}^{*}}{\alpha_{l}} N_{l} .
\end{aligned}
$$

Substituting our choice (4.13) of $\alpha_{l}$ and $\beta_{l}$ we have

$$
N_{l+1}=(l+1) N_{l}
$$

Taking $N_{0}=1$ we conclude

$$
N_{l}=l !
$$

Now, we have the following.

Theorem 5.4 Suppose that $\phi_{l}$ are mode functions on $\left(M, q_{a b}\right)=A \backslash \mathcal{N}$ such that they satisfy the relations (4.14). Multiplying the same constant normalization factor $C$ to all $\phi_{l}, \phi_{l} \rightarrow C \phi_{l}$, does not change those relations. By choosing $C$ appropriately, we can make the normalization

$$
\left(\phi_{l, m, n_{0}}, \phi_{l^{\prime}, m^{\prime}, n_{0}^{\prime}}\right)=l ! \delta_{l l^{\prime}} \delta_{m m^{\prime}} \delta_{n_{0} n_{0}^{\prime}} .
$$

hold.

Proof. The orthogonality for $m$ and $m^{\prime}$ is apparent from the fact that $\mu(m)$ is the eigenvalue of the selfadjoint operator $(1 / i) \chi_{3}$. The orthogonality for $n_{0}$ and $n_{0}^{\prime}$ comes from the orthogonality among the mode functions on $\bar{M}$ :

$$
\left(\bar{\phi}_{l, m, n}, \bar{\phi}_{l, m, n^{\prime}}\right)_{\bar{M}} \equiv \int_{\bar{M}} \bar{\phi}_{l, m, n} \bar{\phi}_{l, m, n^{\prime}}^{*} d \mu_{0}=0, \quad\left(n \neq n^{\prime}\right),
$$


which is also apparent from the fact that the operator $(1 / i) \xi_{2}$, of which eigenvalues are $\nu(n)$, is selfadjoint on $L^{2}(\bar{M})$. Observing that when $n_{0} \neq n_{0}^{\prime}, \phi_{l, m, n_{0}}$ and $\phi_{l, m, n_{0}^{\prime}}$ are linear combinations in different sets $\left\{\bar{\phi}_{l, m, n}\right\}_{n \in \mathcal{N}_{1}}$ and $\left\{\bar{\phi}_{l, m, n}\right\}_{n \in \mathcal{N}_{2}}$, $\mathcal{N}_{1} \cap \mathcal{N}_{2}=\emptyset$, we can easily see

$$
\int_{\bar{M}} \phi_{l, m, n_{0}} \phi_{l, m, n_{0}^{\prime}}^{*} d \mu_{0}=0, \quad\left(n_{0} \neq n_{0}^{\prime}\right)
$$

which in tern implies the orthogonality for $n_{0}$ and $n_{0}^{\prime}$ in $L^{2}(M)$. The other part has already been proven.

Remark. The constant $C$ does not depend on $l$, but can depend on $m$ and $n_{0}$, so we may write $C=C_{m, n_{0}}$.

Apparently, if we define

$$
\phi_{l, m, n_{0}}^{(\mathrm{n})} \equiv \frac{1}{\sqrt{l !}} \phi_{l, m, n_{0}},
$$

they become orthonormal to each other:

$$
\left(\phi_{l, m, n_{0}}^{(\mathrm{n})}, \phi_{l^{\prime}, m^{\prime}, n_{0}^{\prime}}^{(\mathrm{n})}\right)=\delta_{l l^{\prime}} \delta_{m m^{\prime}} \delta_{n_{0} n_{0}^{\prime}} .
$$

As seen from Eqs.(5.13) and (4.9) this corresponds to choosing

$$
\alpha_{l}^{(\mathrm{n})}=e^{i \Theta_{l}} \sqrt{|\mu|(l+1)}, \quad \beta_{l}^{(\mathrm{n})}=-e^{-i \Theta_{l}} \sqrt{|\mu| l},
$$

where $\Theta_{l}$ is an arbitrary phase factor, which we may want to take zero, $\Theta_{l}=0$. Substituting $\alpha_{l}=\alpha_{l}^{(\mathrm{n})}$ and $\beta_{l}=\beta_{l}^{(\mathrm{n})}$ into Eqs. (4.7) we have another version of $\chi$-relations for $\phi_{l, m, n_{0}}^{(\mathrm{n})}$, which have the most direct correspondence to the usual relations between the quantum states of the harmonic oscillator. As mentioned however we employ the unnormalized $\phi_{l}$ in this paper for the convenience of computations.

\section{Further transformation}

The mode functions $\phi_{l, m, n_{0}}(\mathbf{x})$ shown in the previous section are not well defined on the general spacetime solution $(M \times \mathbf{R}, \mathrm{g})=\Gamma \backslash(\tilde{M} \times \mathbf{R}, \tilde{\mathrm{g}})$, because of the fact $A \neq \Gamma$. Remember that $\Gamma \subset G_{\mathrm{II}}$ is a four-parameter embedding, while $A$ is a three-parameter one. This incompatibility means that we cannot identify the coordinates $\mathbf{x}$ in $\mathcal{N}$ with the spatial coordinates $\mathbf{x}$ in $(\tilde{M} \times \mathbf{R}, \tilde{\mathrm{g}})$. 30] Although it is expected that an appropriate diffeomorphism can make the mode functions well defined on the spacetime, such a diffeomorphism can affect the $\chi$-relations. In the following, we show by explicit computations that this is the case but a further renormalization makes the mode functions retain the original $\chi$-relations.

As shown in [18, the covering group $\Gamma$ can be parameterized as

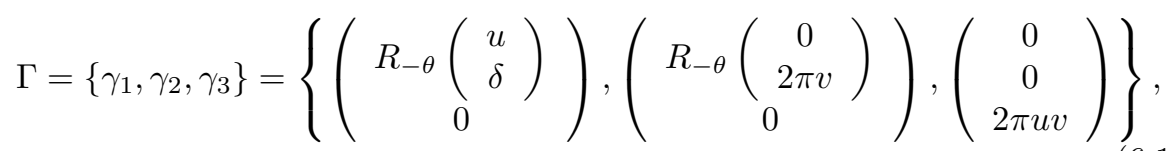


where $\gamma_{i} \in G_{\mathrm{II}}$, and $\theta, u, \delta$, and $v$ are real parameters. 31 32 Let $(M, q)=$ $\Gamma \backslash\left(\tilde{M}, \tilde{q}_{a b}\right)$ be the spatial section of the solution, where $\tilde{q}$ is the spatial part of $\tilde{g}$. For convenience of considering diffeomorphisms between the spatial universal cover $\left(\tilde{M}, \tilde{q}_{a b}\right)$ and the standard conformal manifold $\mathcal{N}=\left(\tilde{M}, e^{2 \alpha} \tilde{q}^{(0)}\right)$, let us distinguish the latter manifold (without metric structure) by denoting $\tilde{M}^{\prime}$. This distinction is meant to be helpful just to keep track of the direction of the diffeomorphisms we consider.

Let $\psi$ be a diffeomorphism:

$$
\psi: \tilde{M} \rightarrow \tilde{M}^{\prime}
$$

such that

$$
\Gamma=\psi^{-1} \circ A \circ \psi
$$

Then, the induced function of $\phi_{l, m, n_{0}}(\mathbf{x})$ will be invariant under $\Gamma$, since so is $\phi_{l, m, n_{0}}(\mathbf{x})$ under $A$.

We can find $\psi$ in $\operatorname{Isom} \mathcal{N}$, which is explicitly given by

$$
\psi=\mathbf{b} \circ s_{\theta},
$$

where $\mathbf{b} \in G_{\mathrm{II}}$ is

$$
\mathbf{b}=\left(\pi v \cos \theta \sin \theta, u^{-1}\left(-\zeta_{-\theta}(u, \delta)+\pi \delta v \sin \theta \cos \theta\right), 0\right) .
$$

We have, for simplicity, chosen the third component of $\mathbf{b}$ as zero, though it can be an arbitrary constant. The induced vectors $\psi^{*} \chi_{I}$ on $\tilde{M}^{\prime}$ becomes a linear transformation of $\chi_{I}$, due to the property that elements in $\operatorname{Isom\mathcal {N}}$ are automorphisms of $G_{\mathrm{II}}$. Note that when acting on the vectors $\chi_{I}$, the induced map $\psi^{*}=\mathbf{b}^{*} \circ s_{\theta}^{*}$ becomes the same as $s_{\theta}^{*}$, since $\chi_{I}$ are by definition invariant under the induced map $\mathbf{b}^{*}$ for $\mathbf{b} \in G_{\mathrm{II}}$. Therefore from (the vector version of) Eq.(2.10), we have

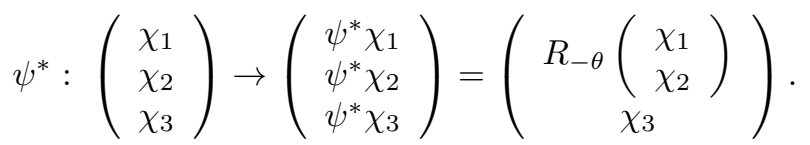

Let $\phi_{l}^{(\mathrm{ss})}(\mathbf{x}) \equiv\left(\psi_{*} \phi_{l}\right)(\mathbf{x})=\phi_{l} \circ \psi(\mathbf{x})$, where $\mathbf{x} \in \tilde{M}$. (Superscript (ss) stands for "spatial section".) Then,

$$
\begin{aligned}
\chi_{1} \phi_{l}^{(\mathrm{ss})} & =\chi_{1}\left(\psi_{*} \phi_{l}\right)=\left(\psi^{*} \chi_{1}\right) \phi_{l} \circ \psi \\
& =\left(\cos \theta \chi_{1}+\sin \theta \chi_{2}\right) \phi_{l} \circ \psi \\
& =\sqrt{\frac{|\mu|}{2}}\left(-e^{-i \theta} \phi_{l+1}+e^{i \theta} l \phi_{l-1}\right) \circ \psi \\
& =\sqrt{\frac{|\mu|}{2}}\left(-e^{-i \theta} \phi_{l+1}^{(\mathrm{ss})}+e^{i \theta} l \phi_{l-1}^{(\mathrm{ss})}\right),
\end{aligned}
$$

where we have used the relations (4.14). Similarly, we obtain

$$
\chi_{2} \phi_{l}^{(\mathrm{ss})}=\varsigma i \sqrt{\frac{|\mu|}{2}}\left(e^{-i \theta} \phi_{l+1}^{(\mathrm{ss})}+e^{i \theta} l \phi_{l-1}^{(\mathrm{ss})}\right),
$$

and $\chi_{3} \phi_{l}^{(\mathrm{ss})}=i \mu \phi_{l}^{(\mathrm{ss})}$. These relations are different from the original $\chi$-relations (4.14) unless $\theta$ is a multiple of $2 \pi$. 
However, it is possible to renormalize $\phi_{l}^{(\mathrm{ss})}$ so that the original $\chi$-relations are recovered. It is straightforward to check that

$$
\varphi_{l}=e^{i \theta l} \phi_{l}^{(\mathrm{ss})}
$$

gives such a renormalized function. Thus, we have found that the functions $\varphi_{l}=e^{i \theta l} \phi_{l} \circ \psi$ with $\phi_{l}$ given by Eq.(5.8) and $\psi$ being Eq. (6.4), are served as the right mode functions on the spatial section (and therefore on the spacetime) that satisfy the relations (4.14). This provides a direct proof of the following:

Theorem 6.1 There exist $|m|$ different sets of time-independent mode functions $\left\{\varphi_{l, m}(\mathbf{x})\right\}_{l=0}^{\infty}$ on the spatially closed Bianchi II solution $(M \times \mathbf{R}, \mathrm{g})=$ $\Gamma \backslash(\tilde{M} \times \mathbf{R}, \tilde{\mathrm{g}})$ such that they satisfy the relations 4.14$)$.

\section{$7 \quad U(1)$-symmetric modes}

Let us, for completeness, consider the modes with the fiber index $m$ being zero. We call these modes $U(1)$-symmetric, since they are constant along the $U(1)$ $\left(\simeq S^{1}\right)$ fibers.

Let $\phi$ be an eigenfunction for $m=0$, i.e., $\chi_{3} \phi=(\partial \phi / \partial z)=0$. This in turn implies that $\chi_{1}$ and $\chi_{2}$ are commutative when acting on $\phi$,

$$
\left[\chi_{1}, \chi_{2}\right] \phi=0,
$$

since $\left[\chi_{1}, \chi_{2}\right]=\chi_{3}$. Due to this property, the harmonics describing the $m=0$ subspace of $L^{2}(M)$ become the usual Fourier expansion on a torus. (Thus, together with the results for the generic modes we obtain Theorem 1.1]) In the following we explicitly determine the spectrum of the eigenvalues for the operators $\chi_{1}$ and $\chi_{2}$ in terms of the spacetime moduli parameters $u, \delta, v$, and $\theta$.

Let us first work on the 3 -manifold $A \backslash \mathcal{N}$ (not on the spacetime manifold) as we did for the generic $(m \neq 0)$ case. Taking the form of $A$ into account, we label the mode functions with the the following equations

$$
\begin{aligned}
\left(u \chi_{1}+\delta \chi_{2}\right) \phi & =2 \pi i k_{1} \phi, \\
v \chi_{2} \phi & =i k_{2} \phi,
\end{aligned}
$$

where the eigenvalues $k_{1}$ and $k_{2}$ are to be used as labels. Let us therefore write the solution of these equations as $\phi=\phi_{k_{1}, k_{2}}^{(0)}(\mathbf{x})=\phi_{k_{1}, k_{2}}^{(0)}(x, y)$, which is given by

$$
\phi_{k_{1}, k_{2}}^{(0)}=\text { constant } \times e^{\frac{i}{u}\left(2 \pi k_{1} x+\frac{k_{2}}{v}(-\delta x+u y)\right)} .
$$

Since, recalling the rule (2.2),

$$
\begin{aligned}
& \phi_{k_{1}, k_{2}}^{(0)}\left(\mathbf{a}_{1} \mathbf{x}\right)=\phi_{k_{1}, k_{2}}^{(0)}(x+u, y+\delta)=\phi_{k_{1}, k_{2}}^{(0)}(\mathbf{x}) e^{2 \pi i k_{1}} \\
& \phi_{k_{1}, k_{2}}^{(0)}\left(\mathbf{a}_{2} \mathbf{x}\right)=\phi_{k_{1}, k_{2}}^{(0)}(x, y+2 \pi v)=\phi_{k_{1}, k_{2}}^{(0)}(\mathbf{x}) e^{2 \pi i k_{2}}
\end{aligned}
$$

we find

$$
k_{1} \in \mathbf{Z}, \quad k_{2} \in \mathbf{Z}
$$


for $\phi_{k_{1}, k_{2}}^{(0)}$ to be well defined on $M=A \backslash \tilde{M}$. The remaining condition $\phi_{k_{1}, k_{2}}^{(0)}\left(\mathbf{a}_{3} \mathbf{x}\right)=$ $\phi_{k_{1}, k_{2}}^{(0)}(\mathbf{x})$ is trivial.

Therefore from Eqs.(7.2) the $\chi$-relations for the $U(1)$-symmetric modes are

$$
\begin{aligned}
& \chi_{1} \phi_{k_{1}, k_{2}}^{(0)}=i K_{1}\left(k_{1}, k_{2}\right) \phi_{k_{1}, k_{2}}^{(0)} \\
& \chi_{2} \phi_{k_{1}, k_{2}}^{(0)}=i K_{2}\left(k_{2}\right) \phi_{k_{1}, k_{2}}^{(0)} \\
& \chi_{3} \phi_{k_{1}, k_{2}}^{(0)}=0,
\end{aligned}
$$

where

$$
\begin{aligned}
K_{1}\left(k_{1}, k_{2}\right) & \equiv \frac{1}{u}\left(2 \pi k_{1}-\frac{\delta}{v} k_{2}\right), \\
K_{2}\left(k_{2}\right) & \equiv \frac{k_{2}}{v} .
\end{aligned}
$$

To extend the mode functions on the spacetime manifold $\Gamma \backslash(\tilde{M} \times \mathbf{R}, \tilde{\mathrm{g}})$, we need to apply the diffeomorphism $\psi$ defined in Eq. (6.4) again, and as a result the spectrum of the eigenvalues are altered. (Contrary to the generic case, any renormalizations of the resulting mode functions do not affect the $\chi$-relations.)

As in the previous section, let us define $\varphi_{k_{1}, k_{2}}^{(0)} \equiv \phi_{k_{1}, k_{2}}^{(0)} \circ \psi$. Then, from Eq.(6.6), we have, e.g.,

$$
\begin{aligned}
\chi_{1} \varphi_{k_{1}, k_{2}}^{(0)} & =\left(\psi^{*} \chi_{1}\right) \phi_{k_{1}, k_{2}}^{(0)} \circ \psi \\
& =\left(\cos \theta \chi_{1}+\sin \theta \chi_{2}\right) \phi_{k_{1}, k_{2}}^{(0)} \circ \psi \\
& =i\left(\cos \theta K_{1}+\sin \theta K_{2}\right) \varphi_{k_{1}, k_{2}}^{(0)} .
\end{aligned}
$$

A similar result is also obtained for $\chi_{2} \varphi_{k_{1}, k_{2}}^{(0)}$. We write the final form of the relations as follows.

$$
\begin{aligned}
& \chi_{1} \varphi_{k_{1}, k_{2}}^{(0)}=i \kappa_{1}\left(k_{1}, k_{2}\right) \varphi_{k_{1}, k_{2}}^{(0)}, \\
& \chi_{2} \varphi_{k_{1}, k_{2}}^{(0)}=i \kappa_{2}\left(k_{1}, k_{2}\right) \varphi_{k_{1}, k_{2}}^{(0)}, \\
& \chi_{3} \varphi_{k_{1}, k_{2}}^{(0)}=0, \quad k_{1} \in \mathbf{Z}, k_{2} \in \mathbf{Z},
\end{aligned}
$$

where

$$
\begin{aligned}
& \kappa_{1}\left(k_{1}, k_{2}\right) \equiv \cos \theta K_{1}\left(k_{1}, k_{2}\right)+\sin \theta K_{2}\left(k_{2}\right), \\
& \kappa_{2}\left(k_{1}, k_{2}\right) \equiv-\sin \theta K_{1}\left(k_{1}, k_{2}\right)+\cos \theta K_{2}\left(k_{2}\right) .
\end{aligned}
$$

Now, we have:

Theorem 7.1 There exist time-independent mode functions $\varphi_{k_{1}, k_{2}}^{(0)}(\mathbf{x}), k_{1}, k_{2} \in$ $\mathbf{Z}$, on the spatially closed Bianchi II solution $(M \times \mathbf{R}, \mathrm{g})=\Gamma \backslash(\tilde{M} \times \mathbf{R}, \tilde{\mathrm{g}})$ such that they satisfy the relations (7.9).

\section{Application to the Klein-Gordon equation}

As an example, let us consider the Klein-Gordon equation

$$
\left(\mathrm{g}^{a b} \nabla_{a} \nabla_{b}-\mathrm{m}_{\Phi}^{2}\right) \Phi=0,
$$


where $\mathrm{m}_{\Phi} \geq 0$ is the mass of the field $\Phi . \nabla_{a}$ is the covariant derivative operator associated with the spacetime metric $\mathrm{g}_{a b}$. It is straightforward to see that this equation on our background can be expressed, using the invariant operators $\chi_{I}$, as

$$
\left(\frac{-1}{\sqrt{-\mathrm{g}}} \frac{\partial}{\partial t}\left(\sqrt{-\mathrm{g}} N^{-2} \frac{\partial}{\partial t}\right)+\triangle_{q}-\mathrm{m}_{\Phi}^{2}\right) \Phi=0,
$$

where $\triangle_{q}$ is the Laplacian with respect to the spatial metric $q_{a b}$;

$$
\triangle_{q}=q_{1}^{-1}\left(\chi_{1}\right)^{2}+q_{2}^{-1}\left(\chi_{2}\right)^{2}+q_{3}^{-1}\left(\chi_{3}\right)^{2},
$$

and $\sqrt{-\mathrm{g}} \equiv \sqrt{-\operatorname{det} \mathrm{g}_{a b}}=4\left|p_{3}\right| \beta t N^{2}$.

Let us consider a generic irreducible component of $\Phi$, i.e., $\Phi=\Phi_{m, n_{0}}, m \neq 0$. We can expand this component as

$$
\Phi(t, \mathbf{x})=\sum_{l=0}^{\infty} a_{l}(t) \varphi_{l}(\mathbf{x}),
$$

where $\varphi_{l}=\varphi_{l, m, n_{0}}$ are the mode functions mentioned in Theorem 6.1

From the relations (4.14), we have

$$
\begin{aligned}
\left(\chi_{1}\right)^{2} \varphi_{l} & =\frac{|\mu|}{2}\left(\varphi_{l+2}-(2 l+1) \varphi_{l}+l(l-1) \varphi_{l-2}\right), \\
\left(\chi_{2}\right)^{2} \varphi_{l} & =-\frac{|\mu|}{2}\left(\varphi_{l+2}+(2 l+1) \varphi_{l}+l(l-1) \varphi_{l-2}\right), \\
\left(\chi_{3}\right)^{2} \varphi_{l} & =-\mu^{2} \varphi_{l},
\end{aligned}
$$

from which we immediately obtain the following wave equations for $a_{l}(t)$ :

$$
\ddot{a}_{l}+\frac{1}{t} \dot{a}_{l}+Z(t) a_{l}=I\left(t ; a_{l-2}, a_{l+2}\right),
$$

where

$$
\begin{aligned}
Z(t) \equiv & \frac{\mu^{2}}{16\left(p_{3}\right)^{2} \beta^{2}}\left(1+\beta^{2} t^{4 p_{3}}\right)^{2} t^{-2 p_{3}}+\mathrm{m}_{\Phi}^{2}\left(1+\beta^{2} t^{4 p_{3}}\right) \\
& +\frac{2 l+1}{2}|\mu|\left(t^{-2 p_{1}}+t^{-2 p_{2}}\right),
\end{aligned}
$$

with the inhomogeneous term $I$ being

$$
I\left(t ; a_{l-2}, a_{l+2}\right) \equiv \frac{|\mu|}{2}\left(t^{-2 p_{1}}-t^{-2 p_{2}}\right)\left(a_{l-2}+(l+2)(l+1) a_{l+2}\right) .
$$

(In $I\left(t ; a_{l-2}, a_{l+2}\right), a_{l-2}$ should be regarded zero when $l=0$ and 1.)

Note that the inhomogeneous term $I$ introduces couplings with the next neighboring modes with $l \pm 2$. The equations (8.6) therefore comprise two systems of infinite number of equations, the one with $l=$ even and the one with $l=$ odd, unless the background is LRS. When on the other hand the background is LRS, each equation (8.6) for a given $l$ becomes closed itself, due to the vanishing of the inhomogeneous term $I$.

When the background is LRS, we can find future $(t \rightarrow \infty)$ asymptotic solutions: 
Proposition 8.1 On the LRS Bianchi II vacuum solution with $p_{1}=p_{2}=$ $2 / 3$ and $p_{3}=-1 / 3$, the scalar field equation (8.6) for a generic mode has the following fundamental solutions as $t \rightarrow \infty$ :

$$
y_{l}^{ \pm}(t)=t^{-\frac{2}{3}} e^{ \pm i \mu T_{\mathrm{KG}}(t)}(1+o(1)),
$$

where

$$
T_{\mathrm{KG}}(t) \equiv \frac{9}{16 \beta} t^{4 / 3}+\frac{\beta \mathrm{m}_{\Phi}^{2}}{\mu^{2}} t^{2 / 3}+\left(\frac{3 \beta}{4}-\frac{8 \beta^{3} \mathrm{~m}_{\Phi}^{4}}{27 \mu^{4}}\right) \log t .
$$

The symbol $o(1)$ stands for a function such that $\lim _{t \rightarrow \infty} o(1)=0$.

Proof. This result is a generalization of Theorem 3.4, Ref. [16], with finite mass $m_{\Phi}$. As emphasized there, it is an appropriate choice of new time variable that is essential to obtain an asymptotic solution. In the present case an appropriate choice $T(t)$ is given by

$$
\frac{d T}{d t}=\frac{3}{4 \beta} t^{1 / 3}+\frac{2 \beta \mathrm{m}_{\Phi}^{2}}{3 \mu^{2}} t^{-1 / 3}+\left(\frac{3 \beta}{4}-\frac{8 \beta^{3} \mathrm{~m}_{\Phi}^{4}}{27 \mu^{4}}\right) \frac{1}{t} .
$$

Following the procedure shown in the reference, one obtains the asymptotic solution (8.9).

It is worth noticing that the asymptotic solution only depends on the fiber index $m$ and the other index $l$ does not affect them. See the final section for more discussion.

\section{Vector harmonics}

Let us discuss how we can construct the vector harmonics.

Fortunately, this is on one hand a trivial issue as the invariant frame $\left\{\sigma^{I}, \chi_{I}\right\}$ is well defined on the compactified manifold $M$ (and on the spacetime manifold $M \times \mathbf{R}$ ). This means we can define the components $T_{I \cdots}{ }^{J \cdots}$ of any sort of tensor $T_{a \ldots}{ }^{b \cdots}$ with respect to this invariant frame;

$$
T_{I \cdots}{ }^{J \cdots}=T_{a \cdots}{ }^{b \cdots} \chi_{I}{ }^{a} \cdots \sigma^{J}{ }_{b} \cdots .
$$

All we have to do is to expand these components with respect to the scalar harmonics $\phi_{l, m, n_{0}}$ and $\phi_{k_{1}, k_{2}}^{(0)}$. This procedure corresponds to using the set of one-forms $\left\{\phi_{l} \sigma^{1}{ }_{a}, \phi_{l} \sigma^{2}{ }_{a}, \phi_{l} \sigma^{3}{ }_{a}\right\}_{l=0}^{\infty}$ as vector harmonics for given $m \neq 0$ and $n_{0}$. (As for $m=0, \phi_{l}$ should of course be replaced by $\phi_{k_{1}, k_{2}}^{(0)}$.) On the spacetime manifold, we also need the timelike mode vectors, as well as the replacement $\phi_{l, m, n_{0}} \rightarrow \varphi_{l, m, n_{0}}$. We therefore can define the following harmonics (basis mode vectors)

$$
\left(V_{l}^{\prime 0}\right)_{a} \equiv \varphi_{l}(d t)_{a},\left(V_{l}^{\prime 1}\right)_{a} \equiv \varphi_{l} \sigma_{a}^{1},\left(V_{l}^{\prime 2}\right)_{a} \equiv \varphi_{l} \sigma_{a}^{2},\left(V_{l}^{\prime 3}\right)_{a} \equiv \varphi_{l} \sigma_{a}^{3},
$$

(or the one with $\varphi_{k_{1}, k_{2}}^{(0)}$ instead of $\varphi_{l}$ for $m=0$ ). We call them the simple vector harmonics.

The advantage of considering these harmonics is that they are apparently complete (since the scalar harmonics used are complete). However, this choice of harmonics does not reflect very well the group structure regarding the rotational automorphisms (2.10). A more natural and convenient choice can be obtained 
by using the "spherical bases" $11 \mathcal{A}_{I}(I=1 \sim 3)$ defined in Eqs. (4.2), or their duals such that $\mathcal{A}_{I}{ }^{a} \varrho^{J}{ }_{a}=\delta_{I}^{J}$;

$$
\varrho^{1} \equiv \frac{1}{\sqrt{2}}\left(\sigma^{1}-\varsigma i \sigma^{2}\right), \quad \varrho^{2} \equiv \frac{1}{\sqrt{2}}\left(\sigma^{1}+\varsigma i \sigma^{2}\right), \quad \varrho^{3} \equiv \varsigma i \sigma^{3} .
$$

Using these we can set up new harmonics as

$$
\left(V_{l}^{0}\right)_{a} \equiv \varphi_{l}(d t)_{a},\left(V_{l}^{1}\right)_{a} \equiv \varphi_{l+1} \varrho^{1}{ }_{a},\left(V_{l}^{2}\right)_{a} \equiv \varphi_{l-1} \varrho^{2}{ }_{a},\left(V_{l}^{3}\right)_{a} \equiv \varphi_{l} \varrho^{3}{ }_{a} .
$$

(When $m=0$ we just use the same harmonics as the simple harmonics.) Beware that $\varphi_{l \pm 1}$ are used to define $\left(V_{l}^{1}\right)_{a}$ and $\left(V_{l}^{2}\right)_{a}$. The reason will become clear below. Because of this index correspondence, we should think that the harmonics $\left\{\left(V_{l}^{I}\right)_{a}\right\}_{I=0}^{3}$ are defined for $l \geq-1$ (not for $\left.l \geq 0\right)$. The basis $\left(V_{-1}^{I}\right)_{a}$ for $l=-1$ is nonzero only for $I=1$, and the others should simply be regarded as zero. We call these harmonics the polarized vector harmonics or the standard vector harmonics.

We can confirm that for given $m$, the simple harmonics and the polarized harmonics span the same space of vector fields. This ensures the completeness of the polarized vector harmonics.

Theorem 9.1 For given fiber index $m \in \mathbf{Z}$ (and given auxiliary index $n_{0}$ ), the linear span of the simple harmonics

$$
\operatorname{Span}\left(V^{\prime m}\right) \equiv\left\{\sum_{l=0}^{\infty} \sum_{I=0}^{3} c_{I, l}\left(V_{l}^{\prime I}\right)_{a} \mid c_{I, l} \in \mathbf{C}\right\}, \quad(m \neq 0)
$$

(the $m=0$ case is defined similarly) and that of the polarized harmonics

$$
\operatorname{Span}\left(V^{m}\right) \equiv\left\{\sum_{l=-1}^{\infty} \sum_{I=0}^{3} c_{I, l}\left(V_{l}^{I}\right)_{a} \mid c_{I, l} \in \mathbf{C}\right\}, \quad(m \neq 0)
$$

(the $m=0$ case is defined similarly) are the same;

$$
\operatorname{Span}\left(V^{\prime m}\right)=\operatorname{Span}\left(V^{m}\right) .
$$

Proof. This is trivial for the $m=0$ case, since in this case the two kinds of harmonics are the same. So, we can assume $m \neq 0$. From the definition (9.4), it is apparent that each basis one-form $V_{l}^{I}$ of the polarized harmonics for given $m$ can be expressed as a linear combination of the simple harmonics belonging to the same $m$. Conversely, each basis one-form $V_{l}^{I I}$ of the simple harmonics for given $m$ can be expressed as a linear combination of the polarized harmonics with the same $m$ as

$$
\begin{aligned}
V_{l}^{\prime 0} & =V_{l}^{0}, \\
V_{l}^{\prime 1} & =\frac{1}{\sqrt{2}}\left(V_{l-1}^{1}+V_{l+1}^{2}\right), \\
V_{l}^{\prime 2} & =\frac{\varsigma i}{\sqrt{2}}\left(V_{l-1}^{1}-V_{l+1}^{2}\right), \\
V_{l}^{\prime 3} & =V_{l}^{3} .
\end{aligned}
$$


Therefore the two sets are related by a regular linear transformation, which proves the identity of the two spans.

The significance of the polarized harmonics is that for given $l$ (and as usual, given $m$ and $n_{0}$ ), the space spanned by them

$$
\operatorname{Span}\left(V_{l}\right) \equiv\left\{\sum_{I=0}^{3} c_{I}\left(V_{l}^{I}\right)_{a} \mid c_{I} \in \mathbf{C}\right\}
$$

is invariant under the operation of $L^{2}$. To show this, let us start with observing the commutation relations

$$
\left[\mathcal{A}_{1}, \mathcal{A}_{2}\right]=\mathcal{A}_{3}, \quad\left[\mathcal{A}_{1}, \mathcal{A}_{3}\right]=0, \quad\left[\mathcal{A}_{2}, \mathcal{A}_{3}\right]=0,
$$

from which one can immediately have

$$
\mathcal{L}_{\mathcal{A}_{I}} \mathcal{A}_{J}=\left[\mathcal{A}_{I}, \mathcal{A}_{J}\right]=\epsilon_{I J} \mathcal{A}_{3},
$$

where $\epsilon_{I J K}$ is the unit skew symmetric symbol; $\epsilon_{123}=+1, \epsilon_{I J K}=\epsilon_{[I J K]}$. Then, noting the duality $\mathcal{A}_{J}{ }^{a} \varrho^{K}{ }_{a}=\delta_{J}^{K}$, it is also easy to see

$$
\mathcal{L}_{\mathcal{A}_{I}} \varrho^{J}=-\delta_{3}^{J} \epsilon_{I K 3} \varrho^{K} .
$$

From this equation and the $\chi$-relations, as well as the identity

$$
\mathrm{L}^{2}=\mathcal{L}_{\mathcal{A}_{1}} \mathcal{L}_{\mathcal{A}_{2}}+\mathcal{L}_{\mathcal{A}_{2}} \mathcal{L}_{\mathcal{A}_{1}}
$$

one obtains

$$
\mathrm{L}^{2}\left(\varphi_{l} \varrho^{I}\right)=-\lambda_{l}^{2} \varphi_{l} \varrho^{I}-2 \sqrt{|\mu|} \delta_{3}^{I}\left(\varphi_{l+1} \varrho^{1}+l \varphi_{l-1} \varrho^{2}\right) .
$$

This equation happens to be valid when $I=0$, as well. Converting to our bases (9.4), we obtain the following statement.

Lemma 9.2 Let $V_{l}^{I}(I=0 \sim 3)$ be the basis mode one-forms defined in Eqs. (9.4), and let $\mathrm{L}^{2}$ be the second order Lie derivative operator defined in Eq. 3.5). Then, it holds that

$$
\begin{aligned}
\mathrm{L}^{2} V_{l}^{0} & =-\lambda_{l}^{2} V_{l}^{0} \\
\mathrm{~L}^{2} V_{l}^{1} & =-\lambda_{l+1}^{2} V_{l}^{1} \\
\mathrm{~L}^{2} V_{l}^{2} & =-\lambda_{l-1}^{2} V_{l}^{2} \\
\mathrm{~L}^{2} V_{l}^{3} & =-\lambda_{l}^{2} V_{l}^{3}-2 \sqrt{|\mu|}\left(V_{l}^{1}+l V_{l}^{2}\right),
\end{aligned}
$$

where $-\lambda_{l}^{2}$ is the eigenvalue of $\mathrm{L}^{2}$ with respect to the mode function $\varphi_{l}$, defined in Eq. 4.15.).

Note that the right hand sides of Eqs. (9.15) are linear combinations of $\left\{V_{l}^{I}\right\}_{I=0}^{3}$ belonging to given $l$. This proves the invariance we claimed:

Theorem 9.3 The linear span $\operatorname{Span}\left(V_{l}\right)$, defined in Eq.(9.9), of the harmonics $\left\{V_{l}^{I}\right\}_{I=0}^{3}$ is invariant under the operation of $\mathrm{L}^{2}$ :

$$
\mathrm{L}^{2} \operatorname{Span}\left(V_{l}\right) \subset \operatorname{Span}\left(V_{l}\right) .
$$


Thanks to this property, if the background spacetime is LRS and the field equation is invariant under the LRS-action induced by $s_{\theta}$ as well as the usual group action, the ODEs reduced from the field equation become all independent from the others. In other words, "each $l$ " decouples from the others in case of LRS. (This property does not occur for the simple harmonics, since the linear span of them for a given $l$ is not $\mathrm{L}^{2}$-invariant.)

Before ending this section let us mention the approach taken in [16, Sec.3.4, which was based on the analogy of the spherically symmetric case [13] or the Bianchi III hyperbolically symmetric case [20]. Let us denote the harmonics used in the reference with two dashes like $\left(V_{l}^{\prime \prime I}\right)_{a} . V_{l}^{\prime \prime 0}$ and $V_{l}^{\prime \prime 3}$ are defined in the same way (up to constant multiplication factor) as the polarized ones;

$$
V_{l}^{\prime \prime 0}=V_{l}^{0}, \quad V_{l}^{\prime \prime 3}=\varsigma \mu V_{l}^{3} .
$$

$V_{l}^{\prime \prime 0}$ is called the time-like basis one-form, while $V_{l}^{\prime \prime 3}$ is called the fiber basis one-form. Let us then consider the "plane field" spanned by $\chi_{1}$ and $\chi_{2}$, which is horizontal to the base manifold. This horizontal plane field is invariant under the natural actions of the fibers generated by $\chi_{3}$. We define the "area two-form" $\varepsilon$ of this field by $\varepsilon_{a b}=2 \sigma^{1}{ }_{[a} \sigma_{b]}^{2}$ (although the plane field is not integrable). We define the horizontal gradient ( $H G$ ) basis one-form (corresponding to the "even" one-form [13, 20]) by taking gradient of the scalar harmonics and subtracting the fiber part;

$$
\begin{aligned}
\left(V_{l}^{\prime \prime 1}\right)_{a} & =\partial_{a} \varphi_{l}-\left(V_{l}^{\prime \prime 3}\right)_{a} \\
& =\left(\chi_{1} \varphi_{l}\right) \sigma^{1}{ }_{a}+\left(\chi_{2} \varphi_{l}\right) \sigma^{2}{ }_{a} \\
& =\sqrt{\frac{|\mu|}{2}}\left(-\left(\varphi_{l+1}-l \varphi_{l-1}\right) \sigma^{1}{ }_{a}+\varsigma i\left(\varphi_{l+1}+l \varphi_{l-1}\right) \sigma^{2}{ }_{a}\right) .
\end{aligned}
$$

And, we define the dual horizontal gradient (DHG) basis one-form (corresponding to the "odd" one-form) by taking the dual gradient associated with $\varepsilon$;

$$
\begin{aligned}
\left(V_{l}^{\prime \prime 2}\right)_{a} & =i \varepsilon_{a}{ }^{b} \partial_{b} \varphi_{l} \\
& =i\left(\left(\chi_{2} \varphi_{l}\right) \sigma^{1}{ }_{a}-\left(\chi_{1} \varphi_{l}\right) \sigma^{2}{ }_{a}\right) \\
& =\sqrt{\frac{|\mu|}{2}}\left(-\varsigma\left(\varphi_{l+1}+l \varphi_{l-1}\right) \sigma^{1}{ }_{a}+i\left(\varphi_{l+1}-l \varphi_{l-1}\right) \sigma^{2}{ }_{a}\right) .
\end{aligned}
$$

To raise an index for $\varepsilon_{a b}$ we use the (inverse of the) standard Bianchi II metric $h^{(0) a b}=\chi_{1}{ }^{a} \chi_{1}{ }^{b}+\chi_{2}{ }^{a} \chi_{2}{ }^{b}+\chi_{3}{ }^{a} \chi_{3}{ }^{b}$. It is clear that the harmonics $V_{l}^{\prime \prime} \equiv$ $\left\{\left(V_{l}^{\prime \prime I}\right)_{a}\right\}_{I=0}^{3}$ are equivalent (i.e., their span is the same) to the polarized harmonics $V_{l} \equiv\left\{\left(V_{l}^{I}\right)_{a}\right\}_{I=0}^{3}$ for each $l$, since they are merely related to each other by a regular linear transformation, as seen from the relations

$$
V_{l}^{\prime \prime 1}=-\sqrt{|\mu|}\left(V_{l}^{1}-l V_{l}^{2}\right), \quad V_{l}^{\prime \prime 2}=-\varsigma \sqrt{|\mu|}\left(V_{l}^{1}+l V_{l}^{2}\right),
$$

as well as (9.17). We call these harmonics the mixed vector harmonics. This choice is particularly convenient for Maxwell's equation, since the $U(1)$-gauge transformation, a shifting of the vector potential by a gradient of scalar, does not affect the component for the DHG basis one-form. (As a result, this component itself becomes gauge-invariant. See the next section.) 


\section{Application to Maxwell's equation}

As an application let us consider the source-free Maxwell equation $\nabla^{a} F_{a b}=0$. Since the electromagnetic field $F_{a b}$ is given by (twice) the exterior derivative of the vector potential $A_{a} ; F_{a b}=\partial_{a} A_{b}-\partial_{b} A_{a}$, this equation can be dealt with by vector harmonics.

Let us consider the irreducible component belonging to given $m \neq 0$ and $n_{0}$. For this component we can expand the vector potential as follows:

$$
A_{a}=\sum_{l=-1}^{\infty} \sum_{I=0}^{3} \gamma_{I}^{(l)}(t)\left(V_{l}^{\prime \prime I}\right)_{a}
$$

where $\left(V_{l}^{\prime \prime I}\right)_{a}$ are the mixed vector harmonics. The four kinds of functions of time $\gamma_{I}^{(l)}(t)(I=0 \sim 3)$ serve as the field variables.

The quantities we are interested in are the $U(1)$-gauge invariant variables, which can be easily found by inspecting components of the field strength $F_{a b}=$ $\partial_{a} A_{b}-\partial_{b} A_{a}$. We obtain the following four independent $U(1)$-invariant variables:

$$
Q_{1}^{(l)}=\gamma_{1}^{(l)}-\gamma_{3}^{(l)}, Q_{2}^{(l)}=\gamma_{2}^{(l)}, P_{1}^{(l)}=\dot{\gamma}_{1}^{(l)}-\gamma_{0}^{(l)}, P_{2}^{(l)}=\dot{\gamma}_{2}^{(l)} .
$$

Although function $P_{3} \equiv \dot{\gamma}_{3}-\gamma_{0}$ is also invariant, it is found that it can be (consistently) solved with the others, due to the constraint part of Maxwell's equation $0=\left(\partial_{t}\right)^{a} \nabla^{b} F_{a b}$, which can be written, using the invariant operator, as

$$
0=N^{-1}\left(q_{1}^{-1} \chi_{1} \dot{A}_{1}+q_{2}^{-1} \chi_{2} \dot{A}_{2}+q_{3}^{-1} \chi_{3} \dot{A}_{3}\right)-\triangle_{q} A_{0},
$$

where $A_{0} \equiv N^{-1}\left(\partial_{t}\right)^{a} A_{a}$, and $A_{I} \equiv \chi_{I}{ }^{a} A_{a}(I=1 \sim 3)$. Laplacian $\triangle_{q}$ is given in Eq. (8.3). The evolution equations $0=\chi_{I}{ }^{a} \nabla^{b} F_{a b}(I=1 \sim 3)$ can similarly be written

$$
\begin{aligned}
0= & -N^{-1} \dot{F}_{0 I}+q_{1}^{-1} \chi_{1} F_{1 I}+q_{2}^{-1} \chi_{2} F_{2 I}+q_{3}^{-1} \chi_{3} F_{3 I}+N^{-1} q_{I}^{-1} \dot{q}_{I} F_{0 I} \\
& -(2 N)^{-1}\left(q_{1}^{-1} \dot{q}_{1}+q_{2}^{-1} \dot{q}_{2}+q_{3}^{-1} \dot{q}_{3}\right) F_{0 I}+\left(q_{1} q_{2}\right)^{-1} q_{3} \delta_{I}^{3} F_{12},
\end{aligned}
$$

(no sum for repeated indices)

where $F_{0 I} \equiv N^{-1}\left(\partial_{t}\right)^{a} \chi_{I}{ }^{b} F_{a b}$ and $F_{I J} \equiv \chi_{I}{ }^{a} \chi_{J}{ }^{b} F_{a b}$. After a rather lengthy computation they become the following:

$$
\begin{aligned}
\dot{Q}_{1}^{(l)}= & P_{1}^{(l)}+\frac{\left(q_{1}^{-1}+q_{2}^{-1}\right) q_{3}}{2|\mu|}\left((2 l+1) P_{1}^{(l)}+\varsigma P_{2}^{(l)}\right)+I_{Q_{1}}, \\
\dot{Q}_{2}^{(l)}= & P_{2}^{(l)}, \\
\dot{P}_{1}^{(l)}= & \left(\frac{\dot{N}}{N}-\frac{1}{2} \frac{\dot{q}_{3}}{q_{3}}\right) P_{1}^{(l)}-\mu^{2} \frac{N^{2}}{q_{3}} Q_{1}^{(l)}+I_{P_{1}}, \\
\dot{P}_{2}^{(l)}= & \left(\frac{\dot{N}}{N}-\frac{1}{2} \frac{\dot{q}_{3}}{q_{3}}\right) P_{2}^{(l)}-\mu^{2} \frac{N^{2}}{q_{3}} Q_{2}^{(l)} \\
& -\frac{\mu}{2} N^{2}\left(q_{1}^{-1}+q_{2}^{-1}\right)\left(Q_{1}^{(l)}+\varsigma(2 l+1) Q_{2}^{(l)}\right)+I_{P_{2}},
\end{aligned}
$$


where the inhomogeneous terms are:

$$
\begin{aligned}
& I_{Q_{1}} \equiv-\frac{\left(q_{1}^{-1}-q_{2}^{-1}\right) q_{3}}{2|\mu|}\left\{(l+2)(l+1)\left(P_{1}^{(l+2)}-\varsigma P_{2}^{(l+2)}\right)\right. \\
& \left.+\left(P_{1}^{(l-2)}+\varsigma P_{2}^{(l-2)}\right)\right\} \\
& I_{P_{1}} \equiv \frac{N^{2}}{4}\left(q_{1}^{-1}-q_{2}^{-1}\right)|\mu|\left\{(l+2)\left(Q_{1}^{(l+2)}+\varsigma(2 l+5) Q_{2}^{(l+2)}\right)\right. \\
& \left.-l^{-1}\left(Q_{1}^{(l-2)}+\varsigma(2 l-3) Q_{2}^{(l-2)}\right)\right\} \\
& -\frac{1}{4}\left(\frac{\dot{q}_{1}}{q_{1}}-\frac{\dot{q}_{2}}{q_{2}}\right)\left\{(l+2)\left(P_{1}^{(l+2)}-\varsigma P_{2}^{(l+2)}\right)\right. \\
& \left.+l^{-1}\left(P_{1}^{(l-2)}+\varsigma P_{2}^{(l-2)}\right)\right\}, \\
& I_{P_{2}} \equiv \frac{N^{2}}{4}\left(q_{1}^{-1}-q_{2}^{-1}\right)|\mu|\left\{(l+2)\left(\varsigma Q_{1}^{(l+2)}+(2 l+5) Q_{2}^{(l+2)}\right)\right. \\
& \left.+l^{-1}\left(\varsigma Q_{1}^{(l-2)}+(2 l-3) Q_{2}^{(l-2)}\right)\right\} \\
& -\frac{1}{4}\left(\frac{\dot{q}_{1}}{q_{1}}-\frac{\dot{q}_{2}}{q_{2}}\right)\left\{(l+2)\left(\varsigma P_{1}^{(l+2)}-P_{2}^{(l+2)}\right)\right. \\
& \left.-l^{-1}\left(\varsigma P_{1}^{(l-2)}+P_{2}^{(l-2)}\right)\right\} \text {. }
\end{aligned}
$$

Again, we can see the same qualitative features in the Klein-Gordon equations; we have two systems of infinite number of equations, the one with $l=$ even and the one with $l=$ odd, unless the background is LRS. When the background is LRS, the couplings between mode $l$ and the next neighbors $l \pm 2$ are cut off due to the vanishing of the inhomogeneous terms $I_{Q_{1}}, I_{P_{1}}$ and $I_{P_{2}}$ and this makes each system of four first-order equations (10.5) for given $l$ closed itself. This is of course a result of the invariance described in Theorem 9.3

\section{Conclusion}

There are three main results about the basic properties for the nilgeometric harmonics obtained in this paper. They are (i) the irreducible decomposition of the regular representation (Theorem [1.1), (ii) the explicit form of the mode functions, and (iii) the differential representation formula, the $\chi$-relations (see Eqs.(4.14) and (7.6)). The decomposition (i) represents the completeness of our harmonics. As for the point (ii), remember that we have two kinds of formula, the one for the canonical manifold and the one for the spacetime manifold. The former is given in Eqs.(5.8) and (7.3), while the latter is obtained by the transformations explicitly given in $\S 6$ and $\S 7$ Remember also that the $\chi$-relations are the most important for the purpose of separation of variables. We also have generalized the (scalar) harmonics to vector harmonics, and demonstrated separation of variables for a scalar equation (the Klein-Gordon equation) and a vector equation (the Maxwell equation). 
As we have seen, when the fiber index $m$ is nonzero the ODEs reduced from a field equation, e.g., the ones from the KG equation, become systems of infinite number of simultaneous equations. In this sense, infinite number of different modes are coupled to each other. This is a result of the fact that the corresponding irreducible representation is infinite dimensional. When the background is LRS however, the couplings between the modes are cut off and as a result, each single reduced KG equation becomes closed itself. Although the Maxwell equations give rise to much more complicated reduced equations because of the multiple components of the field variable, it has the same feature that the couplings between modes disappear when the background is LRS. It is also apparent that the linear perturbation equations will have the same feature if we choose the tensor harmonics so as to possess the invariance under $\mathrm{L}^{2}$ like the vector harmonics do (cf. Theorem 9.3).

An interesting fact is that as shown in $\S 8$ the future asymptotic solution of the LRS KG wave equation depends only on the fiber index $m$ and does not depend on the spin index $l$. This fact seems to indicate a clue for analyzing the generic non-LRS cases, since it suggests that the couplings between the modes asymptotically disappear even when the background is non-LRS, at least if the background is close enough to the LRS one. See [17 for the same ("fiber term dominated") behavior of other models (Bianchi VIII and III). Detailed studies of the non-LRS cases, as well as linear perturbations of the nilgeometric model, will be reported elsewhere, on the basis of this work.

\section{Acknowledgment}

The author wishes to thank Vincent Moncrief for helpful discussions, especially at an early stage of this work. He is also grateful to the organizers, including Akio Hosoya and Tatsuhiko Koike, and the participants of the 5th Workshop on Singularity, Spacetime and Related Physics held at Keio University, Yokohama, December 19-21 2003, for the kind invitation and many beneficial comments.

\section{References}

[1] M. Anderson, Commun. Math. Phys. 222 (2001) 533.

[2] S. Bonanos, Commun. Math. Phys. 22 (1971) 190.

[3] S. Bonanos, Commun. Math. Phys. 26 (1972) 259.

[4] G. F. R. Ellis, and M. A. H. MacCallum, Commun. Math. Phys. 12 (1969) 108.

[5] A. E. Fischer, and V. Moncrief, Class. Quant. Grav. 18 (2001) 4493; and reference therein.

[6] G. B. Folland, "Harmonic analysis in phase space," Ann. of Math. Studies 122, Princeton University Press, Princeton (1989).

[7] J. Hempel, "3-manifolds," Ann. of Math. Studies 86, Princeton University Press, Princeton (1976). 
[8] B. L. Hu, and T. Regge, Phys. Rev. Lett. 29 (1972) 1616; B. L. Hu, J. Math. Phys. 15 (1974) 1748.

[9] R. T. Jantzen, Commun. Math. Phys. 64 (1978) 211.

[10] R. T. Jantzen, J. Math. Phys. 19 (1978), 1163.

[11] R. T. Jantzen, Gauge Invariant Perturbation Theory in Spatially Homogeneous Cosmology, in "Essays in General Relativity: A Festschrift for Abraham Taub," ed. F.J. Tipler, Academic Press (1980), 97.

[12] T. Koike, M. Tanimoto, and A. Hosoya, J. Math. Phys. 35 (1994) 4855.

[13] T. Regge and J. A. Wheeler, Phys. Rev. 108, (1957) 1063.

[14] P. Scott, Bull. London Math. Soc. 15 (1983) 401.

[15] M. Sugiura "Unitary Representations and Harmonic Analysis," Second edition, North-Holland/Kodansha, Amsterdam (1990).

[16] M. Tanimoto, Contemp. Math. 337, "Recent Advances in Riemannian and Lorentzian Geometries", AMS (2003), 171. (gr-qc/0306087)

[17] M. Tanimoto, preprint.

[18] M. Tanimoto, T. Koike, and A. Hosoya, J. Math. Phys. 38 (1997) 350.

[19] M. Tanimoto, T. Koike, and A. Hosoya, J. Math. Phys. 38 (1997) 6560.

[20] M. Tanimoto, V. Moncrief, and K. Yasuno, Class. Quant. Grav. 20 (2003) 1879.

[21] A. H. Taub, Ann. Math. 53 (1952) 472.

[22] M. E. Taylor, "Noncommutative Harmonic Analysis" (Mathematical Surveys and Monographs vol. 22), American Mathematical Society, Providence, (1986).

[23] W. P. Thurston, "Three-Dimensional Geometry and Topology Vol.1," Princeton University Press, Princeton (1997).

[24] R. M. Wald, "General Relativity," The University of Chicago Press, Chicago, (1984).

[25] The "left" regular representation can also be defined similarly. We take the "right" one for the reason explained below.

[26] In [12, 18, 19, the second component of $\mathbf{a}_{1}$ was taken zero instead of the first component of $\mathbf{a}_{2}$. It is however not difficult to see that those two choices are equivalent (conjugate) to each other following the procedure shown in the same references. The present parameterization will be proved more convenient for our purpose.

[27] While Isom $\mathcal{N}$ is four-dimensional, the extra isometries are not necessary for us to represent $\mathbf{a}_{i}$, resulting in $\mathbf{a}_{i} \in G_{\mathrm{II}}$ here. Remark however that to obtain this representation the fourth dimensional isometries play an essential role, especially to delete the first component of $\mathbf{a}_{2}$. 
[28] Like this, we consider any functions on $M$ or $\bar{M}$ as functions on the universal cover $\tilde{M}=\mathbf{R}^{3}$. If $f(A x)=f(x)$ holds we regard $f(x)$ as a function on $M=A \backslash \tilde{M}$. Similarly, If $f(\bar{A} x)=f(x)$ holds, $f(x)$ is a function on $\bar{M}=\bar{A} \backslash \tilde{M}$. Those functions are called automorphic functions.

[29] We could impose a unitary condition on $\mathcal{A}_{i}(i=1,2)$ to make the choice of $\alpha_{l}$ and $\beta_{l}$ unique, although there is no reason to do so for our purpose. Our present choice (4.10) makes the final formula (see Eqs.(4.14)) to some extent simpler, with less 'square roots' appearing in the formula than in the one obtained requiring the unitary condition. It is however easy to convert the resulting mode functions into those for unitary $\mathcal{A}_{i}$. See the last paragraph of $\S[$

[30] We are assuming that the action of $A$ on the spacetime manifold $\tilde{M} \times \mathbf{R}$ is defined by

$$
A \circ(\mathbf{x}, t)=(A \circ \mathbf{x}, t), \quad(\mathbf{x}, t) \in \tilde{M} \times \mathbf{R} .
$$

(Apparently, the same equality for $\Gamma$ defines its action on $\tilde{M}$.)

[31] The parameterization among the free parameters has been rearranged from [18] so that it is convenient for our purpose.

[32] Although we use, for convenience, the same symbols $\theta, u, \delta$, and $v$ as in $A$ here, it is worth emphasizing that their meaning is distinctly different; Those in $\Gamma$ are moduli parameters of spacetime, while those in $A$ are the Teichmüller (or moduli) parameters of space, which are functions of time in spacetime [18, 19]. The spacetime moduli parameters parameterize the admissible initial values and velocities of the Teichmüller parameters. 